\title{
THE FIRST GALAXIES: ASSEMBLY UNDER RADIATIVE FEEDBACK FROM THE FIRST STARS
}

\author{
Andreas H. Pawlik ${ }^{1,2}$, Miloš Milosa Vljević ${ }^{1}$, and VolKer Bromm ${ }^{1}$ \\ ${ }^{1}$ Department of Astronomy and Texas Cosmology Center, The University of Texas at Austin, TX 78712, USA \\ ${ }^{2}$ Max Planck Institute for Astrophysics, Karl-Schwarzschild-Str. 1, D-85748 Garching, Germany \\ Received 2012 August 17; accepted 2013 February 23; published 2013 March 25
}

\begin{abstract}
We investigate how radiative feedback from the first stars affects the assembly of the first dwarf galaxies. To this end, we perform cosmological zoomed smoothed particle hydrodynamics simulations of a dwarf galaxy assembling inside a halo reaching a virial mass $\sim 10^{9} M_{\odot}$ at $z=10$. The simulations follow the non-equilibrium chemistry and cooling of primordial gas and the subsequent conversion of the cool dense gas into massive metal-free stars. To quantify the radiative feedback, we compare a simulation in which stars emit both molecular hydrogen dissociating and hydrogen/helium ionizing radiation with a simulation in which stars emit only molecular hydrogen dissociating radiation, and further with a simulation in which stars remain dark. Photodissociation and photoionization exert a strong negative feedback on the assembly of the galaxy inside the main minihalo progenitor. Gas condensation is strongly impeded, and star formation is strongly suppressed in comparison with the simulation in which stars remain dark. The feedback on the gas from either dissociating or ionizing radiation implies a suppression of the central dark matter densities in the minihalo progenitor by factors of up to a few, which is a significant deviation from the singular isothermal density profile characterizing the dark matter distribution inside the virial radius in the absence of radiative feedback. The evolution of gas densities, star formation rates, and the distribution of dark matter becomes insensitive to the inclusion of dissociating radiation in the late stages of the minihalo assembly, and it becomes insensitive to the inclusion of ionizing radiation once the minihalo turns into an atomically cooling galaxy. The formation of a rotationally supported extended disk inside the dwarf galaxy is a robust outcome of our simulations not affected by the inclusion of radiation. Low-mass galaxies in the neighborhood of the dwarf galaxy show a large scatter in the baryon fraction which is driven by radiative feedback from sources both internal and external to these galaxies. Our estimates of the observability of the first galaxies show that dwarf galaxies such as simulated here will be among the faintest galaxies the upcoming James Webb Space Telescope will detect. Our conclusions regarding the structure and observability of the first galaxies are subject to our neglect of feedback from supernovae and chemical enrichment as well as to statistical uncertainties implied by the limited number of galaxies in our simulations.
\end{abstract}

Key words: cosmology: theory - galaxies: formation - galaxies: high-redshift - hydrodynamics - radiative transfer - stars: formation

Online-only material: color figures

\section{INTRODUCTION}

The birth of star-forming galaxies a few hundred million years after the big bang marks an important milestone in the history of our universe. The spectacular images returned by the Hubble Space Telescope (HST) in the past few years have already allowed us to probe into the first billion years of the universe. The last few years have also seen the development of an observationally testable theory of the formation of the first galaxies. As ongoing and upcoming observations, such as with the James Webb Space Telescope (JWST), are about to push to ever earlier times, approaching the epoch of the very first galaxies, this theory is put to ever more stringent tests (for a review see, e.g., Dunlop 2012).

Both analytical arguments (e.g., Tegmark et al. 1997; Naoz et al. 2006; Tseliakhovich et al. 2011) and simulations (e.g., Abel et al. 2002; Bromm et al. 2002; Stacy et al. 2011) suggest that the first stars have formed at $z \gtrsim 30$ inside dark matter minihalos with virial temperatures $\gtrsim 10^{3} \mathrm{~K}$, corresponding to halo masses $\sim 10^{5}-10^{6} M_{\odot}$ (for a review, see Bromm \& Larson 2004). The metal-free primordial gas inside these minihalos cools and condenses to reach the high densities needed to form stars primarily through the radiative de-excitation of rovibrationally excited molecular hydrogen (e.g., Haiman et al. 1996b; Abel et al. 1997). As the minihalos grow in mass, their virial temperatures increase and, after reaching $\sim 10^{4} \mathrm{~K}$, become sufficiently large to collisionally excite atomic hydrogen. This gives birth to the first atomically cooling galaxies with typical masses $\sim 10^{7}-10^{8} M_{\odot}$ at $z \gtrsim 15$ (e.g., Oh \& Haiman 2002; Wise \& Abel 2007b; Greif et al. 2008; for a review see Bromm \& Yoshida 2011). The first atomically cooling galaxies then evolve into the first dwarf galaxies with characteristic masses $\gtrsim 10^{9} M_{\odot}$ (e.g., Mashchenko et al. 2008; Wise et al. 2012). The aim of the current work is to investigate the assembly of such galaxies under the radiative feedback from the first stars. The mass scale marked by the first dwarf galaxies is closely related to a number of key open issues, some of which are outlined below.

Suppression of star formation by radiative feedback. The radiation emitted by the first stars has a profound effect on subsequently forming stars and galaxies (for a comprehensive overview, see Ciardi \& Ferrara 2005). Radiation in the Lyman-Werner (LW) bands dissociates molecular hydrogen, the main coolant in minihalos (e.g., Haiman et al. 1997). Hydrogenionizing radiation heats the gas inside the first halos and the intergalactic medium (IGM). The associated increase in pressure drives the gas outside halos with virial temperatures $\lesssim 10^{4} \mathrm{~K}$, suppressing star formation in both minihalos and the first atomic 
cooling halos (e.g., Thoul \& Weinberg 1996; Barkana \& Loeb 1999). The increased pressure in the IGM impedes the accretion of gas onto these low-mass halos, an effect known as Jeans filtering (e.g., Shapiro et al. 1994; Gnedin \& Hui 1998; Okamoto et al. 2008). In contrast, star formation inside the first dwarf galaxies should be more robust to this negative radiative feedback as their deeper gravitational potentials allow them to hold on to their gas more strongly.

The sources of reionization. The first galaxies are thought to have started the reionization of the universe, which is the transformation of the cosmic hydrogen from its early neutral to its present ionized state that occurred during the first billion years after the big bang (for reviews see, e.g., Barkana \& Loeb 2001; Furlanetto et al. 2006; Meiksin 2009). However, whether galaxies could sustain reionization and drive it to completion is a question of significant debate (e.g., Bouwens et al. 2012; Finkelstein et al. 2012). The largest uncertainties are related to our poor knowledge of the escape fraction, i.e., the fraction of ionizing photons that leave the galaxies unabsorbed and are thus available to reionize the IGM, and of the abundance and ionizing luminosities of low-mass galaxies too faint to be detected in current surveys (e.g., Robertson et al. 2010). The most recent determinations of the UV luminosity density at $z \gtrsim 7$ suggest that a significant contribution from faint, yet to be observed, low-mass galaxies is likely needed to sustain reionization in the recombining gas (Finkelstein et al. 2012; see Kuhlen \& FaucherGiguère 2012 for a comprehensive discussion). Because of their increased robustness against stellar feedback, dwarf galaxies are among the low-mass galaxies expected to be especially efficient sources of reionization (e.g., Choudhury \& Ferrara 2007; Raičević et al. 2011).

The origin of the Milky Way $(M W)$ satellites. Dissipationless simulations of dark matter subhalos around MW-like galaxies imply the existence of a large population of satellite galaxies, only a few of which are currently observed. A number of solutions have been offered to explain this missing satellite problem (Moore et al. 1999; Klypin et al. 1999; for reviews see, e.g., Bullock 2010; Ricotti 2010; Mayer 2010), including the suppression of star formation in low-mass halos by stellar feedback and reionization (e.g., Ricotti \& Gnedin 2005; Salvadori \& Ferrara 2009; Bovill \& Ricotti 2009; Sawala et al. 2012; Brooks \& Zolotov 2012), the transformation of low-mass halos by tidal forces and ram-pressure stripping upon their entry in the MW virial region (e.g., Mayer et al. 2007), modifications of the cold dark matter structure formation paradigm (e.g., Lovell et al. 2012), and others (e.g., Bovy \& Dvorkin 2012; Vera-Ciro et al. 2013; Wang et al. 2012). However, current theories still struggle to explain the abundance and properties of the observed MW satellites across the luminosity range, from the recently discovered ultra-faint to the long-known classical satellites (e.g., Strigari et al. 2008; Bovill \& Ricotti 2011; Boylan-Kolchin et al. 2011). The most massive of the simulated MW dark matter subhalos have progenitors with masses $10^{8}-10^{10} M_{\odot}$ at $z \gtrsim 6$ (e.g., Boylan-Kolchin et al. 2012). This suggests an intimate relation with the first dwarf galaxies, and renders investigations into these objects a promising tool to understand the origin of structure in galaxies such as the MW.

The first disk galaxies. Simulations of the first atomically cooling galaxies, i.e., galaxies inside halos with masses $10^{7}-10^{8} M_{\odot}$ at redshifts $z \sim 15$, reveal a highly irregular morphology of the halo gas (e.g., Wise \& Abel 2007b; Greif et al. 2008; Regan \& Haehnelt 2009). On the other hand, simulations of galaxies inside halos with larger masses $\gtrsim 10^{9} M_{\odot}$ and at lower redshifts $z \lesssim 6-10$ often find the halo gas organized in rotationally supported disks (e.g., Mashchenko et al. 2008; Pawlik et al. 2011; Romano-Díaz et al. 2011; Wise et al. 2012). These findings suggest a dwarf-size mass scale $\sim 10^{8}-10^{10} M_{\odot}$ for the transition to disk-like morphologies, and the emergence of the first disk galaxies at $z \gtrsim 6$. Physical processes to imprint such a scale include the turbulence generated by the cold inflow of gas along filaments which characterizes gas accretion by the first atomic cooling halos (e.g., Wise \& Abel 2007b; Wise et al. 2008; Greif et al. 2008), and stellar feedback (e.g., Kaufmann et al. 2007). Whether the first halos may host disks is an important open issue, affecting estimates of, e.g., the escape of ionizing photons into the IGM (e.g., Gnedin et al. 2008; Conroy $\&$ Kratter 2012), or the ability of massive black holes to accrete gas and grow (e.g., Eisenstein \& Loeb 1995; Koushiappas et al. 2004; Lodato \& Natarajan 2006; Petri et al. 2012).

The faintest galaxies JWST will see. The faintest $z \gtrsim 6$ galaxies HST has so far revealed have estimated stellar masses $\gtrsim 10^{8} M_{\odot}$ (Labbé et al. 2010; Finkelstein et al. 2010; Curtis-Lake et al. 2013). Future observations with upcoming telescopes such as the JWST will allow to search for galaxies down to still lower stellar masses and out to higher redshifts, thus promising to test our theories of the formation of the first stars and galaxies. However, most studies agree that even JWST will not be sensitive enough to detect the stellar radiation emitted from inside the minihalos and the first atomic cooling halos (e.g., Haiman \& Loeb 1998; Oh et al. 2001; Ricotti et al. 2008; Johnson et al. 2009; Zackrisson et al. 2012; Rydberg et al. 2013). JWST may detect the stellar radiation from some of these objects if they are gravitational lensed (e.g., Zackrisson et al. 2012). But most of the stellar light collected by JWST from high redshifts is expected to come from dwarf galaxies more massive than the first atomically cooling galaxies (e.g., Johnson et al. 2009; Pawlik et al. 2011).

Motivated primarily by the exciting prospects for observations with the upcoming JWST, we have previously presented cosmological simulations of a dwarf galaxy assembling inside a halo reaching $10^{9} M_{\odot}$ at $z=10$ (Pawlik et al. 2011). The simulations were performed using the smoothed particle hydrodynamics (SPH) technique and achieved high resolution by zooming in a select region around the galaxy. Following the non-equilibrium chemistry and cooling of primordial gas, the simulations tracked the evolution of the dwarf galaxy starting from before its birth inside a minihalo. An intriguing outcome was the formation of a rotationally supported extended disk just prior to the final simulation redshift. However, our previous simulations did not account for star formation and the associated feedback. As explained above, stellar feedback has the potential to significantly affect the assembly of the gas inside low-mass halos.

In this study we present a new set of simulations similar to our previous simulations, but extending them by including star formation and radiation. We focus on the radiative feedback from LW and ionizing radiation. ${ }^{3}$ To judge the impact of radiative feedback, we will compare a simulation that includes both LW and ionizing radiation with a simulation that includes only LW radiation and further with a simulation in which no radiation is emitted. Note that the simulations do not account for supernova (SN) feedback or metal enrichment, a limitation we will discuss in Section 7. The simulations are designed

\footnotetext{
We will use the terms LW radiation and dissociating radiation interchangeably.
} 
primarily to address the impact of radiative feedback on the assembly of the emerging dwarf galaxy, and on the formation of galactic disks inside it. However, we will also briefly discuss the impact of radiative feedback on the assembly of galaxies in the neighborhood of the simulated dwarf galaxy. Our simulations enable us to provide an improved estimate of the observability of the first galaxies with JWST.

The organization of this paper is as follows. In Section 2 (as well as in the Appendix) we describe our numerical techniques, and in Section 3 we describe the set of simulations that we have carried out. In Sections 4 and 5, we present the results of our simulations, subsequently discussing the assembly of the dwarf galaxy and the radiative feedback on the IGM and the neighboring galaxies. In Section 6, we use the simulated star formation rates (SFRs) to estimate the observability of the first galaxies with JWST. In Section 7, we discuss our results and also address some of the most important limitations. In Section 8, we summarize our work.

Throughout this work we assume the $\Lambda$ CDM cosmological model with parameters $\Omega_{\mathrm{m}}=0.258, \Omega_{\mathrm{b}}=0.0441, \Omega_{\Lambda}=$ $0.742, \sigma_{8}=0.796, n_{\mathrm{s}}=0.963$, and $h=0.719$, which are consistent with the most recent analysis of the observations with the Wilkinson Microwave Anisotropy Probe satellite (Komatsu et al. 2011). Distances are expressed in physical (i.e., not comoving) units, unless noted otherwise. We will make use of the species number density fractions with respect to hydrogen $\eta_{\alpha} \equiv n_{\alpha} / n_{\mathrm{H}}$, where $\alpha$ labels the chemical species.

\section{NUMERICAL METHODS}

In this section we describe the numerical techniques employed. The simulations presented below are identical to the simulations described in Pawlik et al. (2011), except for the inclusion of star formation and dissociating and ionizing radiation. We will therefore only briefly review the simulation techniques already used in Pawlik et al. (2011). We will focus on the description of the techniques used to model the formation of stars and the dissociative and ionizing impact of stellar radiation on the gas. We remind the reader that the simulations do not account for SN feedback or metal enrichment, as discussed further in Section 7.

\subsection{Gravity and Hydrodynamics}

We use a modified version of the $N$-body/TreePM SPH code GADGET (Springel 2005; Springel et al. 2001b; Schaye et al. 2010) to perform a suite of zoomed cosmological hydrodynamical simulations of the assembly of a halo that reaches a virial mass $M_{\text {vir }} \approx 10^{9} M_{\odot}$ at redshift $z=10$. The simulations are initialized at redshift $z=127$ in a box of size 3.125 $h^{-1} \mathrm{Mpc}$ comoving. Initial particle positions and velocities are obtained by applying the Zeldovich approximation (Zeldovich 1970) to particles arranged on a Cartesian grid. We adopt a transfer function for matter perturbations generated with CMBFAST (version 4.1; Seljak \& Zaldarriaga 1996).

We first perform a low-resolution simulation without star formation down to redshift $z=10$, and locate the most massive halo, with virial mass $M_{\mathrm{vir}} \approx 10^{9} M_{\odot}$ and virial radius $r_{\text {vir }}=3.1 \mathrm{kpc}$. We then trace the particles found within $3 r_{\text {vir }}$ from the most bound particle of this halo back to their locations at the start of the simulation. We hierarchically refine the initial particle setup using a nested sequence of cubical patches ("zooms") centered on the traced particles, inside which we increase the mass resolution by successive factors of eight with each increasing level of zoom. In the zoom with the highest mass resolution, which entirely contains the traced particles and which we refer to as the refinement region, gas (dark matter) particles have masses $m_{\mathrm{g}}=484 M_{\odot}\left(m_{\mathrm{DM}}=\right.$ $\left.2350 M_{\odot}\right)$. The simulations are performed with the gravitational forces softened over a sphere of Plummer-equivalent radius $\epsilon=0.1 h^{-1} \mathrm{kpc}$ comoving applied to all particles.

The particle dynamical variables, such as position, velocity, and density, are evolved in time using individual particle gravito-hydrodynamical time steps determined by the smaller of the dynamical time step and the Courant time step (e.g., Equation (16) in Springel 2005), which is the standard GADGET time stepping scheme. We do not explicitly limit the particle gravito-hydrodynamical time steps by either the chemical time or the radiative cooling or radiative heating time. However, chemistry, radiative cooling, and radiative heating described below are solved by subcycling the smallest gravitohydrodynamical time step among all particles in the simulation on the relevant timescales (see Appendix A.1.4).

\subsection{Chemistry and Cooling}

We assume that the gas is of primordial composition with hydrogen mass fraction $X=0.75$ and a helium mass fraction $Y=1-X$. We use a modified version of the implicit solver DVODE (Brown et al. 1989) to follow the non-equilibrium chemistry and cooling of $\mathrm{H}_{2}, \mathrm{D}, \mathrm{HD}, \mathrm{D}^{+}, \mathrm{H}^{+}, \mathrm{H}, \mathrm{D}$, and $\mathrm{He}$, and we include $\mathrm{H}^{-}$and $\mathrm{H}_{2}^{+}$assuming their collisional equilibrium abundances (Johnson \& Bromm 2006; Greif et al. 2010). We consider all relevant radiative cooling processes: cooling by collisional ionization, collisional excitation of atomic and molecular lines, the emission of free-free and recombination radiation, and Compton cooling by the cosmic microwave background. Once stars form and emit radiation, the chemical and thermal evolution of the gas is also affected by the photodissociation of molecular hydrogen and deuterium, and photoionization of hydrogen and helium, as we describe in Sections 2.5 and 2.6. It should be kept in mind that at high gas densities, a Jeans floor employed to avoid artificial fragmentation artificially increases the gas temperature and affects the distribution and dynamics of the gas (see Equation (1) in Pawlik et al. 2011).

\subsection{Star Formation}

Star formation is a complex astrophysical phenomenon, many details of which remain to be understood (for a review see, e.g., McKee \& Ostriker 2007). In the MW and in nearby galaxies, star formation is observed to occur inside a hierarchy of clouds with masses $\sim 10^{4}-10^{6} M_{\odot}$. The clouds are transformed into stars at a rate $\dot{\rho}_{\star}=\rho_{\mathrm{cl}} / \tau_{\star}$, where $\tau_{\star}=\tau_{\mathrm{ff}} / \epsilon_{\mathrm{ff}}$ is the star formation timescale, $\tau_{\mathrm{ff}}=\left[3 \pi /\left(32 G \rho_{\mathrm{cl}}\right)\right]^{1 / 2}$ is the free-fall time at the characteristic density $\rho_{\mathrm{cl}}$ of the star-forming clouds, and $\epsilon_{\mathrm{ff}}$ is the star formation efficiency per free-fall time. Star formation is observed to be a slow process with a typical efficiency per free-fall time of only $\epsilon_{\mathrm{ff}} \sim 0.01$, independent of the characteristic densities of the star-forming clouds in the range $n_{\mathrm{H}, \mathrm{cl}} \equiv \rho_{\mathrm{cl}} X / m_{\mathrm{H}} \sim 10-10^{5} \mathrm{~cm}^{-3}$ (Krumholz \& Tan 2007).

While gas masses $\sim 10^{4}-10^{6} M_{\odot}$ that characterize starforming clouds in the nearby universe are resolved, our simulations lack the resolution and physical detail to follow the formation of individual stars. Hence, we cannot exploit our simulations to estimate the SFRs inside individual star-forming clouds from first principles. Instead, we adopt a phenomenological model that specifies how quickly gas turns into stars. The 
model is motivated by and consistent with investigations of star formation in the nearby universe. Prescriptions for treating star formation in simulations of high-redshift galaxies are commonly calibrated with relations obtained from the local universe. This practice is necessitated by the current lack of direct observations of star formation at high redshifts. The phenomenological approach is sufficient to allow us to investigate the effect of stellar dissociating and photoionizing radiation on the interstellar gas and the IGM.

We restrict star formation to occur only in regions with gas densities exceeding a threshold density, $n_{\mathrm{H}} \geqslant n_{\mathrm{SF}}$, where we set $n_{\mathrm{SF}} \equiv 500 \mathrm{~cm}^{-3}$. This is somewhat lower than the densities $\sim 10^{4} \mathrm{~cm}^{-3}$ of metal-free clouds on the verge of collapse to form stars (e.g., Bromm et al. 2002; Abel et al. 2002). It is also lower than the similar densities of metal-free clouds able to shield their $\mathrm{H}_{2}$ from external dissociating radiation (e.g., SafranekShrader et al. 2012). Because our simulations focus on the assembly of galaxies inside more massive halos, we must adopt, for reasons of computational viability, a lower resolution than employed in these previous works, preventing us from adopting still larger star formation threshold densities. Our choice for the star formation threshold density is close to the lowest threshold density $n_{\mathrm{H}}=1000 \mathrm{~cm}^{-3}$ explored by Muratov et al. (2012) above which the time of the formation of the first star formed inside high-redshift minihalos was found to be insensitive to an increase in the threshold density (see also, e.g., Maio et al. 2009; Wise et al. 2012).

We adopt a star formation timescale that depends solely on the threshold density for star formation $n_{\mathrm{SF}}$,

$$
\tau_{\star} \equiv \frac{\tau_{\mathrm{ff}}\left(n_{\mathrm{SF}}\right)}{\epsilon_{\mathrm{ff}}}=201.2 \mathrm{Myr}\left(\frac{n_{\mathrm{SF}}}{500 \mathrm{~cm}^{-3}}\right)^{-1 / 2},
$$

where we have set $\epsilon_{\mathrm{ff}}=0.01$. Hence, the star formation timescale is independent of the gas density $n_{\mathrm{H}} \geqslant n_{\mathrm{SF}}$. This amounts to assuming that all star formation occurs inside clouds with characteristic density $n_{\mathrm{H}, \mathrm{cl}}=n_{\mathrm{SF}}$, and is consistent with both our limited resolution and the observations in the nearby universe. A similar star formation recipe using a density-independent star formation time has been employed in, e.g., Kravtsov (2003). However, we caution that the SFRs of simulated galaxies depend on the specific choice for the value of the star formation efficiency, at least unless star formation is self-regulated by feedback (e.g., Ricotti et al. 2002; Haas et al. 2012).

Our numerical implementation of the star formation law is identical to that of Schaye \& Dalla Vecchia (2008). The star formation law is interpreted stochastically, and the probability that a star-forming gas particle is turned into a star particle in a time interval $\Delta t$ is given by $\min \left(\Delta t / \tau_{\star}, 1\right)$. Gas particles are converted to star particles assuming a conversion efficiency of $100 \%$, i.e., the masses of the star particles are identical to those of the gas particles from which they are formed. The implied relatively large mass of star particles is consistent with our resolution, but sets a lower limit on the stellar mass fractions and hence ionizing and LW luminosities in the simulated galaxies to which the effects of radiative feedback may be sensitive. We impose an upper limit $T_{\star} \equiv \max \left(1.5 \times 10^{4} \mathrm{~K}, 10^{0.5} \times T_{\text {floor }}\right)$ on the temperature at which gas is allowed to form stars, where $T_{\text {floor }}$ is the minimum temperature set by the Jeans floor.

\subsection{Population Synthesis}

We interpret the star particles in our simulations as simple stellar populations, i.e., instantaneous stellar bursts that are characterized by an initial mass function (IMF), metallicity, and age. We compute the time-dependent hydrogen and helium ionizing luminosities $Q_{\mathrm{HI}}, Q_{\mathrm{He} \text { I }}$, and $Q_{\mathrm{He} \text { II }}$, as well as the luminosities $Q_{\mathrm{LW}}$ in the LW band with energies $11.2-13.6 \mathrm{eV}$, of these star formation bursts using the population synthesis models from Schaerer (2003). The models assume a powerlaw IMF $p\left(m_{\star}\right) \propto m_{\star}^{-\alpha}$ with the Salpeter (1955) exponent $\alpha=2.35$ but allow for a variation of the range of the stellar masses sampled from the power-law distribution. We describe the stellar bursts using the Schaerer (2003) zero-metallicity models with initial masses in the range $50-500 M_{\odot}$. The age of a burst is the time difference between the simulation time at which the star particle was created and the current simulation time. For reference, the luminosities at zero-age main sequence are $Q_{\mathrm{HI}}=10^{47.98}, Q_{\mathrm{He}}=10^{47.80}, Q_{\mathrm{He} \text { II }}=10^{47.05}$, and $Q_{\mathrm{LW}}=$ $10^{46.96}$ photons $M_{\odot}^{-1} \mathrm{~s}^{-1}$. We only consider star particles inside the refinement region, and we do not follow the propagation of radiation outside this region.

\subsection{Photodissociation}

Molecular hydrogen $\mathrm{H}_{2}$ and deuterated hydrogen $\mathrm{HD}$ are photodissociated upon absorption of radiation in the LW band belonging to the photon energy range $11.2-13.6 \mathrm{eV}$. The rate of photodissociation of $\mathrm{H}_{2}$ is (e.g., Abel et al. 1997)

$$
\begin{aligned}
k_{\mathrm{LW}} & =1.1 \times 10^{8} \mathrm{~s}^{-1} \frac{F_{\nu}\left(v_{\mathrm{LW}}\right)}{\mathrm{erg} \mathrm{Hz}^{-1} \mathrm{~s}^{-1} \mathrm{~cm}^{-2}} \\
& =1.38 \times 10^{-12} \mathrm{~s}^{-1} J_{21},
\end{aligned}
$$

where $\nu_{\mathrm{LW}} \equiv 12.4 \mathrm{eV}$ is the characteristic LW frequency, $F_{v}\left(\nu_{\mathrm{LW}}\right)$ is the LW flux, $J_{21}=J_{\nu} /\left(10^{-21} \mathrm{erg} \mathrm{Hz}^{-1} \mathrm{~s}^{-1} \mathrm{~cm}^{-2} \mathrm{sr}^{-1}\right)$ is the normalized LW mean intensity, and $J_{v}=F_{v}\left(v_{\mathrm{LW}}\right) / 4 \pi$. We set the rate for photodissociation of HD identical to that of $\mathrm{H}_{2}$ (e.g., Glover \& Jappsen 2007; Wolcott-Green \& Haiman 2011).

We write the LW intensity $J_{21}$ as the sum of the cosmological LW background intensity $\bar{J}_{21}$ and the local LW intensity $J_{21}^{\text {loc }}$ produced by the stellar populations represented by the star particles in the refinement region of our simulations, $J_{21}=$ $\bar{J}_{21}+J_{21}^{\text {loc }}$. The refinement region is too small to follow the buildup of the cosmological LW background in a self-consistent manner. We therefore treat the intensity of the LW background as a free parameter, and approximate its evolution using

$$
\bar{J}_{21}(z)=\bar{J}_{21,0} \times 10^{-\left(z-z_{0}\right) / 5},
$$

where $\bar{J}_{21,0}$ is the intensity of the LW background at $z_{0}$. Setting $\bar{J}_{21,0}=1$ and $z_{0}=10$, Equation (3) provides a good fit to the LW background evolution presented in Figure 1 of Greif \& Bromm (2006), and is also consistent with computations of the LW background in other works (e.g., Wise \& Abel 2005; Ahn et al. 2009). The use of an LW background that is a fixed function of redshift does not allow us to capture the effects of self-regulation of star formation inside minihalos by LW feedback (e.g., Ahn et al. 2012), at least until the intensity of LW radiation emitted by the stars inside the simulation box has become higher than the intensity of the background.

For reasons of computational efficiency, we determine the contributions from the $N_{\star}$ star particles in the refinement region to the intensity $J_{i}^{\text {loc }}=\sum_{j=1}^{N_{\star}} J_{j}^{\star}$ evaluated at the location of gas particle $i$ in the optically thin approximation (e.g., Wise \& Abel 
2008a), i.e.,

$$
J_{j}^{\star}=\frac{h_{\mathrm{P}} \nu_{\mathrm{LW}}}{4 \pi \delta \nu_{\mathrm{LW}}} \frac{Q_{\mathrm{LW}, j} m_{j}^{\star}}{4 \pi r_{i j}^{2}}
$$

or, in units of $10^{-21} \mathrm{erg} \mathrm{Hz}^{-1} \mathrm{~s}^{-1} \mathrm{~cm}^{-2} \mathrm{sr}^{-1}$,

$$
J_{21, j}^{\star} \approx 1\left(\frac{Q_{\mathrm{LW}, j}}{10^{47} M_{\odot}^{-1} \mathrm{~s}^{-1}}\right)\left(\frac{m_{j}^{\star}}{500 M_{\odot}}\right)\left(\frac{r_{i j}}{1 \mathrm{kpc}}\right)^{-2} .
$$

Here, $Q_{\mathrm{LW}, j}$ is the photon luminosity of star particle $j$ per unit mass in the LW frequency band, $\delta v_{\mathrm{LW}}=(13.6 \mathrm{eV}-11.2 \mathrm{eV}) / h_{\mathrm{P}}$ is the width of the LW band, $h_{\mathrm{P}}$ is Planck's constant, $m_{j}^{\star}$ is the mass of star particle $j$, and $r_{i j}$ is the distance between the gas and the star particle.

For simplicity, we approximate the time-dependent LW luminosities $Q_{\mathrm{LW}}$ computed in Section 2.4 by their zero-age main-sequence values, and we assume that the stars emit LW radiation for $3 \mathrm{Myr}$ (Schaerer 2003). We approximately account for radiative transfer (RT) effects by attenuating the total LW intensity $J_{21}$ by a local self-shielding factor (Equation (10) in Wolcott-Green et al. 2011 with $\alpha=1.1$ ). The self-shielding factor depends on the column density of molecular hydrogen, which we compute in the local Jeans approximation (e.g., Shang et al. 2010).

When computing the contribution to the LW intensity from local sources using Equation (4), we ignore the absorption of LW radiation along the way to the absorbing gas particle. Near emitters, this is justified because molecular hydrogen is efficiently collisionally dissociated inside the H II regions surrounding them (Johnson et al. 2007; but see Ricotti et al. 2002). Outside the HII regions, this approximation is good as long as the intergalactic molecular hydrogen fraction does not substantially exceed its primordial value, $\eta_{\mathrm{H}_{2}} \sim 10^{-6}$. In this case, the optical depth for absorption in the LW band remains insignificant out to distances $\gtrsim 1 \mathrm{Mpc}$ comoving (e.g., Ricotti et al. 2001, their Figure 12; see also Glover \& Brand 2003, their Figure 7; Ahn et al. 2009), much larger than the size of the refinement region. Finally, we also ignore the absorption of LW photons by atomic hydrogen series lines, which also is not significant on the small scales simulated here (e.g., Haiman et al. 2000; Ahn et al. 2009). Our implementation of photodissociation is similar to that in Johnson et al. (2013), but we do not account for the photo-detachment of $\mathrm{H}^{-}$.

\subsection{Photoionization}

In this section, we describe our implementation of photoionization by stellar radiation. This implementation involves two main steps. First, we transport ionizing photons radially from the star particles through the simulation box, and compute the fraction of the photons absorbed by atomic hydrogen and helium. Second, we infer the associated photoionization and photoheating rates. Here we will only give a brief overview. We present a detailed description of the implementation of the RT and the computation of the photoionization and photoheating rates in the Appendix. There we will also discuss tests of this implementation. The coupling of the RT with the hydrodynamical evolution is achieved by passing the photoionization and photoheating rates, along with the photodissociation rates described in the previous section, to the non-equilibrium solver for the chemical and thermal evolution of the gas described in Section 2.2, and this coupling is described in Appendix A.1.4.
We transport the ionizing radiation emitted by the star particles using the multi-frequency RT code TRAPHIC (Pawlik \& Schaye 2008, 2011). TRAPHIC solves the time-dependent RT equation by tracing photon packets emitted by source particles at the speed of light and in a photon-conserving manner through the simulation box. The photon packets are transported directly on the spatially adaptive, unstructured grid traced out by the SPH particles, which allows one to exploit the full dynamic range of the SPH simulations. A directed radial transport of the photon packets from the sources is accomplished despite the irregular distribution of SPH particles by guiding the photon packets inside cones. A photon packet merging technique renders the computational cost of the RT independent of the number of ionizing sources. The transport of photons is discretized in RT time steps $\Delta t_{\mathrm{r}}$, after each of which the chemical and thermal evolution of the gas is advanced based on the number of absorbed photons.

We make the following approximations specific to the current work. Each star particle emits ionizing photon packets to its neighboring SPH particles once per RT time step in a set of tessellating emission cones centered around eight different directions. The effective angular sampling of the surrounding volume is larger than implied by this number of directions thanks to the splitting of photon packets among neighbors inside the same emission cone, and because of the randomization of the emission directions at each RT time step. The photons are transported radially away from the star particles by tracing them downstream inside transmission cones with solid angle $4 \pi / 128$. This angular resolution is sufficiently high to track the delay of the ionization fronts around individual halos by dense filaments, giving rise to the typical "butterfly" shape of ionized regions, as shown in Figure 1.

To reduce the computational cost, we limit the propagation of photons to at most a single inter-particle distance per RT time step, which approximates the full time-dependent RT in the limit of small RT time steps. Ionizing photons are transported using a single frequency bin, and the absorption of the photons by neutral hydrogen and neutral and singly ionized helium is computed in the gray approximation. The gray approximation does not allow us to capture effects of spectral hardening, and hence we may underestimate the effects of photoionization and photoheating near and ahead of ionization fronts, such as the enhanced formation of molecular hydrogen (Ricotti et al. 2001, 2002). These approximations are discussed in further detail in the Appendix, in particular in Appendix A.2.3.

\section{SIMULATIONS}

We employ a set of three simulations to study the effects of radiative feedback on the assembly of high-redshift galaxies. Simulation $L W+R T$ includes both star formation and the $L W$ and ionizing radiation emitted by the stars, as well as an imposed LW radiation background, as described in Section 2. We will focus on discussing results of this simulation. We will often present our results by comparing this simulation with simulation $L W$, which is identical except that the emission of ionizing radiation is disabled, and with simulation $N O F B$, in which the emission of LW radiation is also disabled. In the last simulation, gas forms stars, but these stars do not emit radiation, and in addition, the intensity of the assumed LW background is set to zero. This simulation is therefore identical to simulation $Z 4$ reported in Pawlik et al. (2011), except for the inclusion of star formation. Important parameters of the simulations presented here are summarized in Table 1. The final redshift of the simulations 

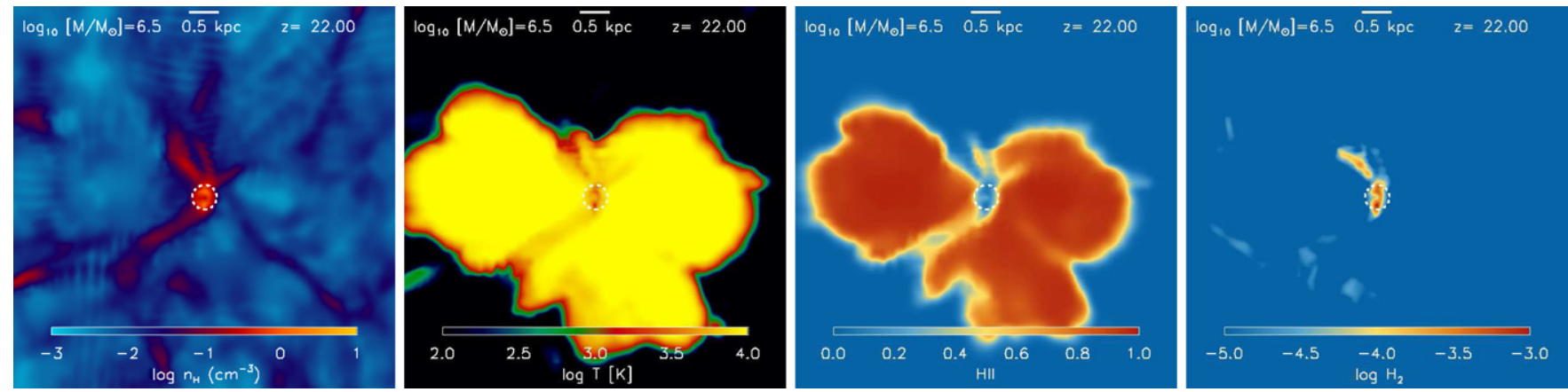

Figure 1. Gas density, temperature, ionized hydrogen fraction, and molecular hydrogen fraction (from left to right) averaged in a thin slice of thickness $0.2 \mathrm{kpc}$ through the center of the minihalo progenitor at $z=22$, shortly after the first stellar burst has shut off. The relic photoionized region of diameter 4-6 kpc shows the typical non-spherical "butterfly" shape (e.g., Abel et al. 1999). The dense gas inside the virial radius and along the filaments cools rapidly, allowing the residual-free electrons to catalyze the formation of molecular hydrogen up to fractions $\eta_{\mathrm{H}_{2}} \sim 2 \times 10^{-3}$ (Oh \& Haiman 2002). The molecular hydrogen fraction is also increased near the transition to the predominantly neutral IGM, a relict of the increased rate of molecular hydrogen formation inside the ionization fronts (Ricotti et al. 2002). The dashed circle marks the virial radius. For purpose of visualization, this figure has been obtained from a simulation identical to simulation $L W+R T$ but with an increased angular sampling, in which the propagation of photons was not limited to a single inter-particle distance. In Appendix A.2.3, we discuss that this change in parameters leaves the evolution of the minihalo essentially unaffected.

(A color version of this figure is available in the online journal.)

is $z=11$. We note that simulations $L W$ and $N O F B$ are identical to the simulations used to estimate the detectability of pair instability SNe in Hummel et al. (2012).

We use the friends-of-friends (FOF) halo finder, with linking parameter $b=0.2$, built into the substructure finder SUBFIND (Springel et al. 2001a), to extract halos from our simulations. Given an FOF halo, we use SUBFIND to identify its most bound particle and let it mark the halo center. We then obtain the virial radius, defined as the radius of the sphere centered on the most bound particle within which the average matter density is equal to 200 times the redshift-dependent critical density of the universe. The total mass inside this sphere defines the halo virial mass. We define the total SFR of a given halo as the sum of the SFRs of the gas particles it contains. Because we employ a stochastic star formation recipe, the rate at which star-forming gas particles are converted to star particles may randomly fluctuate around this SFR.

We make use of the following relation between virial temperature $T_{\text {vir }}$ and virial mass $M_{\text {vir }}$ (e.g., Equation (3.12) in Loeb 2010; see also Barkana \& Loeb 2001),

$$
T_{\mathrm{vir}}=1.04 \times 10^{4} \mathrm{~K}\left(\frac{M_{\mathrm{vir}}}{10^{8} M_{\odot}}\right)^{2 / 3}\left(\frac{\mu}{0.6}\right)\left(\frac{1+z}{10}\right) .
$$

\section{FORMATION AND EVOLUTION OF THE DWARF GALAXY}

In this section, we present the formation history of the simulated dwarf galaxy. We start with discussing the radiative feedback from LW and ionizing radiation on the assembly of gas and the formation of stars inside the dark matter halo hosting the galaxy (Section 4.1), and on the assembly of the dark matter halo (Section 4.2). We finally proceed to investigate the robustness of the gaseous disks forming at the center of the dwarf galaxy halo under the radiative feedback (Section 4.3).

\subsection{Baryon Assembly in the Dwarf Galaxy Halo}

Figure 2 shows the formation history of the dwarf galaxy in simulation $L W+R T$, including both photodissociating and photoionizing radiation (blue curves). For comparison, the figure also shows the formation histories of the dwarf galaxy in the simulation in which star particles were sources of LW
Table 1

Simulation Parameters

\begin{tabular}{lcccccc}
\hline \hline Simulation & $m_{\mathrm{gas}}{ }^{\mathrm{a}}$ & $m_{\mathrm{DM}}{ }^{\mathrm{b}}$ & $\epsilon^{\mathrm{c}}$ & $n_{\mathrm{SF}}{ }^{\mathrm{d}}$ & $\mathrm{LW}^{\mathrm{e}}$ & $\mathrm{RT}^{\mathrm{f}}$ \\
\hline$L W+R T$ & $4.84 \times 10^{2}$ & $2.35 \times 10^{3}$ & 0.1 & 500 & Yes & Yes \\
LW & $4.84 \times 10^{2}$ & $2.35 \times 10^{3}$ & 0.1 & 500 & Yes & No \\
NOFB & $4.84 \times 10^{2}$ & $2.35 \times 10^{3}$ & 0.1 & 500 & No & No \\
\hline
\end{tabular}

Notes.

${ }^{\mathrm{a}}$ Gas particle mass in the refinement region $\left(M_{\odot}\right)$.

${ }^{\mathrm{b}}$ Dark matter particle mass in the refinement region $\left(M_{\odot}\right)$.

${ }^{\mathrm{c}}$ Gravitational softening radius ( $h^{-1} \mathrm{kpc}$ comoving).

${ }^{\mathrm{d}}$ Star formation threshold density $n_{\mathrm{SF}}\left(\mathrm{cm}^{-3}\right)$.

e Photodissociation by stellar LW photons and the LW background.

${ }^{\mathrm{f}}$ Radiative transfer of stellar ionizing photons.

but not of photoionizing radiation ( $L W$; red curves), and in the simulation in which star particles remained dark and the intensity of the LW background was set to zero (NOFB; black curves). The formation history is obtained by using SUBFIND to locate the dwarf halo progenitor that contains most of the 50 most bound particles of the dwarf halo at the final simulation redshift $z=11$, and then repeating this procedure to find the progenitor of this progenitor and so on, tracing the halo assembly back to $z=25$. The quantities displayed in Figure 2 are obtained from the properties of the particles inside the virial radius. The figure shows that the dark matter halo hosting the emerging dwarf galaxy grows by about three orders of magnitude in mass in about 300 million years, consistent with expectations (Pawlik et al. 2011, their Figure 1).

The evolution of the galaxy in simulation $L W+R T$ proceeds in several main phases which will be discussed below: (1) the assembly of a dark matter minihalo with mass $\sim 10^{6} M_{\odot}$ and the accretion and condensation of gas inside it, leading to the formation of stars just below $z=23$, (2) the subsequent accretion of gas under feedback from star formation inside the dark matter minihalo, (3) the evolution of this minihalo into an atomically cooling halo at $z \approx 16$, during which the properties of the galaxy become insensitive to the inclusion of LW radiation, (4) the ensuing growth into a dwarf halo, during which the properties of the galaxy become robust against feedback from photoionization, and, finally, (5) the formation of two nested rotationally supported gaseous disks below $z \lesssim$ 

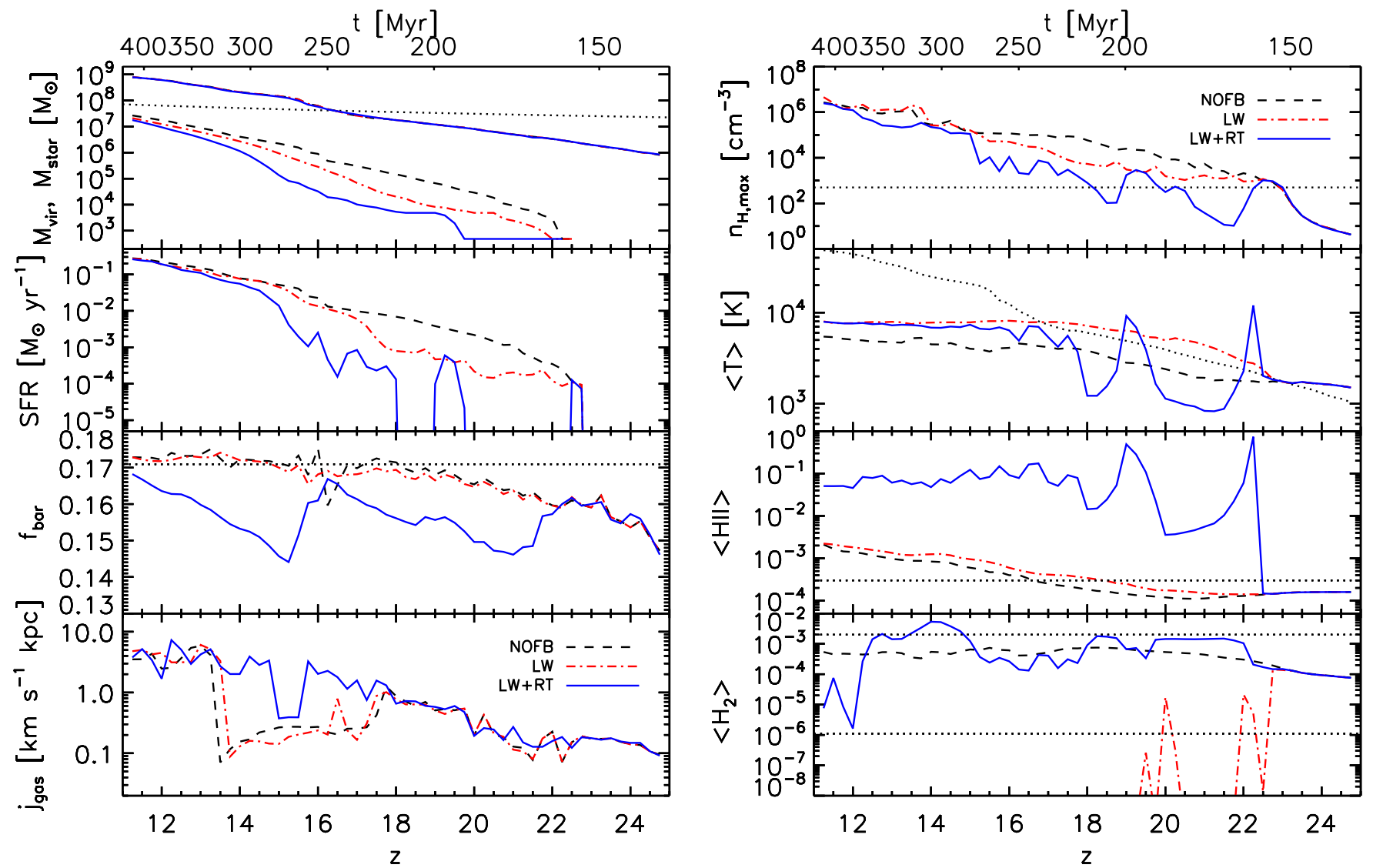

Figure 2. Assembly history of the dwarf galaxy. The blue solid curves in each panel show the evolution of the indicated property of the dwarf galaxy in simulation $L W+R T$ which included both $L W$ and ionizing radiation. For comparison, the red dash-dotted and the black dashed curves show, respectively, the evolution of the same properties in simulation $L W$ that included only LW but not ionizing radiation and in simulation NOFB in which star particles remained dark and the intensity of the LW background was set to zero. The left-hand panel shows, from top to bottom, (a) virial mass $M_{\text {vir }}$ (upper set of three curves which are nearly on top of each other) and stellar mass $M_{\star}$ (lower set of three curves), and the mass corresponding to a virial temperature $T_{\mathrm{vir}}=10^{4} \mathrm{~K}$ (dotted; Equation (6) with $\mu=0.6$ ), (b) SFR, (c) baryon fraction, and the universal baryon fraction $\Omega_{\mathrm{b}} / \Omega_{\mathrm{m}} \approx 0.17$ (dotted), and (d) specific angular momentum $j_{\text {gas }}$ of the gas. The right-hand panel shows, from top to bottom, (a) the maximum gas density $n_{\mathrm{H}, \max }$, (b) the mass-weighted mean temperature $\langle T\rangle$, and the virial temperature corresponding to the virial mass of the dwarf galaxy (in the NOFB simulation, dotted), (c) the mass-weighted mean ionized hydrogen fraction $\left\langle\eta_{\mathrm{H}}\right\rangle_{\mathrm{I}}$, and the initial ionized hydrogen fraction, $\eta_{\mathrm{H} \text { II }}=3 \times 10^{-4}$ (dotted), and (d) the mass-weighted mean molecular hydrogen fraction $\left\langle\eta_{\mathrm{H}_{2}}\right\rangle$, the initial molecular hydrogen fraction, $\eta_{\mathrm{H}_{2}}=1.1 \times 10^{-6}$ (lower dotted), and the freeze-out molecular fraction expected in fossil $\mathrm{H}$ II regions, $\eta_{\mathrm{H}_{2}} \approx 2 \times 10^{-3}$ (upper dotted; Oh \& Haiman 2002). All quantities are computed considering only matter inside the redshift-dependent virial radius $r_{\mathrm{vir}}$. The inclusion of photodissociation and photoheating implies a strong negative feedback on the condensation of gas and the formation of stars in the minihalo progenitor, but has little effect after the minihalo evolved into an atomically cooling galaxy.

(A color version of this figure is available in the online journal.)

13.5. Our discussion of these phases will be complemented by comparisons with the evolution of the dwarf galaxy in simulations $L W$ and $N O F B$.

During the first phase, and as the dark matter halo grows in mass and accretes gas, both the baryon fraction, which is initially slightly smaller than the cosmic baryon fraction $\Omega_{\mathrm{b}} / \Omega_{\mathrm{m}} \approx 0.17$, and the central gas densities increase. By $z=25$, the molecular hydrogen fraction has significantly departed from its initial value, enabling the minihalo gas to cool efficiently. The average gas temperature inside the virial radius is initially slightly higher than the virial temperature (dotted curve). Note that in simulation $N O F B$, as the molecular hydrogen fraction builds up, this relation then reverses and the virial temperature becomes higher than the average gas temperature (e.g., O'shea \& Norman 2007). At $z \approx 23$, the central gas densities become larger than the threshold density for star formation. A single gas particle is turned into a star particle, triggering the emission of LW and ionizing radiation from the associated stellar burst. At that time, the halo has reached a mass of $\sim 2 \times 10^{6} M_{\odot}$.

Photoionization from the radiation emitted by the first stellar burst almost instantly increases the average gas temperatures to $\gtrsim 10^{4} \mathrm{~K}$. The associated increase in thermal pressure pushes the gas away from the minihalo center, and removes a fraction of it from inside the virial radius. As a consequence, the baryon fraction is reduced to about $f_{\text {bar }}=0.15$. The reduction in the baryon fraction is consistent with but slightly smaller than that found in previous simulations of the assembly of minihalos under feedback from star formation (e.g., Wise \& Abel 2008a; Wise et al. 2012). This may be because the minihalo simulated here is fed by dense filaments, and the inflow of gas along these filaments provides a strong obstacle for photoheating to drive the gas beyond the virial radius, and it replenishes the photoevaporated regions with fresh gas (e.g., Abel et al. 2007). Nevertheless, the reduction in the central gas mass is sufficient to drive the central gas densities below the threshold density for star formation, and the stellar burst shuts itself off. Figure 1 shows images of the gas density, temperature, and ionized and molecular hydrogen fraction around the minihalo at the end of the stellar burst.

After the first stellar burst is shut off, the average massweighted molecular hydrogen fraction approaches $\sim 2 \times 10^{-3}$, as expected inside the relic H II region (Oh \& Haiman 2002). The increased fraction of molecular hydrogen enables the gas to cool quickly, and the central gas densities increase. A few 
tens of Myr after the end of the first burst, the gas has become sufficiently cold and dense for another stellar burst to be ignited (e.g., O'shea et al. 2005; Alvarez et al. 2006). Photoionization heating from this second burst again lowers the gas densities, but this time there is no strong decrease in the baryon fraction. The accreting minihalo is thus massive enough to retain most of the gas inside the virial region. Because the negative feedback from photoheating is now less strong, this second starburst is more extended in time than the first one, and it involves the conversion of several gas particles to star particles. However, the combined feedback from the stellar clusters represented by the star particles eventually shuts off star formation. But already a few tens of Myr later the central gas densities have again increased above the SF threshold density, and the galaxy continues to form stars.

By redshift $z \approx 16$, the halo has reached virial temperatures $T_{\text {vir }} \gtrsim 10^{4} \mathrm{~K}$. Consequently, a significant fraction of the atomic hydrogen is collisionally excited, and its radiative de-excitation endows the gas with an additional channel to lose its thermal energy. Feedback from photoionization heating continues to keep the gas inside the halo at relatively low densities. However, the galaxy now forms stars continuously, albeit at a rate significantly smaller than in the absence of radiative feedback. The comparison with the quickly rising SFRs in simulation $L W$ that included emission of LW radiation but not that of ionizing photons shows that the feedback from the photodissociation of molecular hydrogen by LW radiation alone becomes inefficient in preventing the gas from forming stars as the halo mass approaches the atomic cooling limit, in good agreement with previous works (e.g., Ahn \& Shapiro 2007; Wise \& Abel 2007a; O'shea \& Norman 2008). The transformation of the minihalo into an atomic cooling halo is accompanied by a significant decrease of the baryon fraction by $\approx 20 \%$ at $z \approx 15-16$ and in the specific angular momentum of the gas $j_{\text {gas }} \equiv J_{\text {gas }} / M_{\text {gas }}$, where $J_{\text {gas }}$ is the total gas angular momentum with respect to the motion of the most bound particle and $M_{\text {gas }}$ the total gas mass.

Below $z \lesssim 15$, photoionization heating becomes increasingly inefficient at reducing the density of the halo gas, and the gas then condenses up to $\gtrsim 10^{5} \mathrm{~cm}^{-3}$. As a result, the expansion of $\mathrm{H}$ II regions is impeded by the increased recombination rates. The properties of the galaxy then become rapidly insensitive to the inclusion of ionizing radiation. The average massweighted ionized fraction, which has been increasing until then, remains roughly constant at around $\left\langle\eta_{\mathrm{H}}{ }_{\text {II }}\right\rangle \lesssim 0.1$. As a result of the large densities, the SFRs approach those seen in the simulation without radiation. The average mass-weighted molecular hydrogen fraction reaches values in excess of $\left\langle\eta_{\mathrm{H}_{2}}\right\rangle \gtrsim$ $2 \times 10^{-3}$ as $\mathrm{H}_{2}$ forms efficiently not only in fossil $\mathrm{H}$ II regions (e.g., Oh \& Haiman 2002; Johnson et al. 2007) but also in the ionization fronts of the $\mathrm{H}$ II regions under irradiation from the ionizing stars (Ricotti et al. 2002; see also Shapiro \& Kang 1987; Jeon et al. 2012).

A major merger at redshift $z \approx 15$ is accompanied by a strong increase in the specific angular momentum of the halo gas. The gas then settles in a disk, which is essentially in place at $z \approx 13.5$. The merger and the formation of this disk are shown in Figure 4, which display a sequence of snapshots of the gas density inside the forming dwarf galaxy. The disk is surrounded by a second larger-scale disk at $z \approx 12$. The evolution and the properties of these two disks will be discussed in more detail in Section 4.3. After the formation of the disks, the difference between virial and average gas temperatures that has amplified since the halo has become an atomic cooling halo continues to increase. In the atomic cooling halo this difference arises primarily because a fraction of the gas does not shock-heat to the virial temperature upon accretion onto the halo. Instead, the gas can efficiently cool inside the dense filaments that reach into the virial region and supply the halo center with gas (Pawlik et al. 2011). A qualitatively similar difference between average and virial temperatures has been seen in other simulations of highredshift low-mass galaxies (e.g., O'shea \& Norman 2007; Wise $\&$ Abel 2007b; Greif et al. 2008). At $z=11$, the final simulation redshift, the SFRs reach $\sim 0.2 M_{\odot} \mathrm{yr}^{-1}$, consistent with the SFRs found in previous simulations of high-redshift low-mass galaxies (e.g., Wise \& Cen 2009; Razoumov \& Sommer-Larsen 2010; Yajima et al. 2011; Wise et al. 2012).

\subsection{Effect of Radiative Feedback on the Dark Matter Halo}

Radiative feedback also affects the properties of the dark matter halo hosting the emerging dwarf galaxy. Figure 3 compares the evolution of the dark matter density profile of the galaxy in simulation $N O F B$ with that in simulation $L W+R T$ (left panel), and with that in simulation $L W$ (right panel). The bottom panels show the dark matter density profiles for each pair of simulations at four representative redshifts. The profiles are scaled by dividing by a singular isothermal profile $\rho_{\text {iso }}(r)=\rho_{\text {vir }} r_{\text {vir }}^{2} / r^{2}$. In the last expression, $r_{\text {vir }}$ is the virial radius of the halo, $\rho_{\text {vir }}=200 \rho_{\text {crit }}$, and $\rho_{\text {crit }}$ is the critical density. The top panels show the ratios of the density profiles of the simulations including radiation and the simulation without radiation shown in the bottom panels, which helps to illustrate the effects of stellar feedback. In simulation $N O F B$, i.e., in the absence of radiative feedback (dashed curves), the dark matter density profile is approximately singular isothermal at all redshifts.

The central dark matter densities in the simulations that included ionizing and/or LW radiation (solid curves in each of the bottom panels) are initially significantly lower, by up to factors $\sim 5$, than those in the simulation without radiation. The dark matter density profiles in these simulations thus do not follow a singular isothermal shape but show a spatially resolved dark matter "core," extending to radii significantly larger than the gravitational softening scale (leftmost vertical lines). The reduction in the central dark matter densities is more distinct and exists down to lower redshifts in simulation $L W+R T$ than in simulation $L W$. The difference in the central dark matter densities originates in the difference in the distribution of the gas inside the assembling dwarf galaxy. In simulation $L W$, gas cannot cool and condense as efficiently as in simulation $N O F B$ because molecular hydrogen, the main coolant in low-mass primordial galaxies, is photodissociated by LW radiation. In simulation $L W+R T$, the central gas densities are, on average, further reduced as photoionization heating drives the gas away from the halo center. The radiative feedback on the distribution of baryons implies a significant change in the gravitational potential and, in turn, in the gravitational pull on the dark matter, which hence remains less centrally concentrated.

The comparison of the dark matter density profiles in the simulations with and without feedback demonstrates that the ability of gas to cool and condense to high densities is crucial for establishing the singular isothermal density profile seen in the simulation without feedback (e.g., Wise \& Abel 2008b; Zemp et al. 2012). That gas condensation can lead to a more concentrated dark matter density distribution is well known and is usually described using the framework of halo contraction 

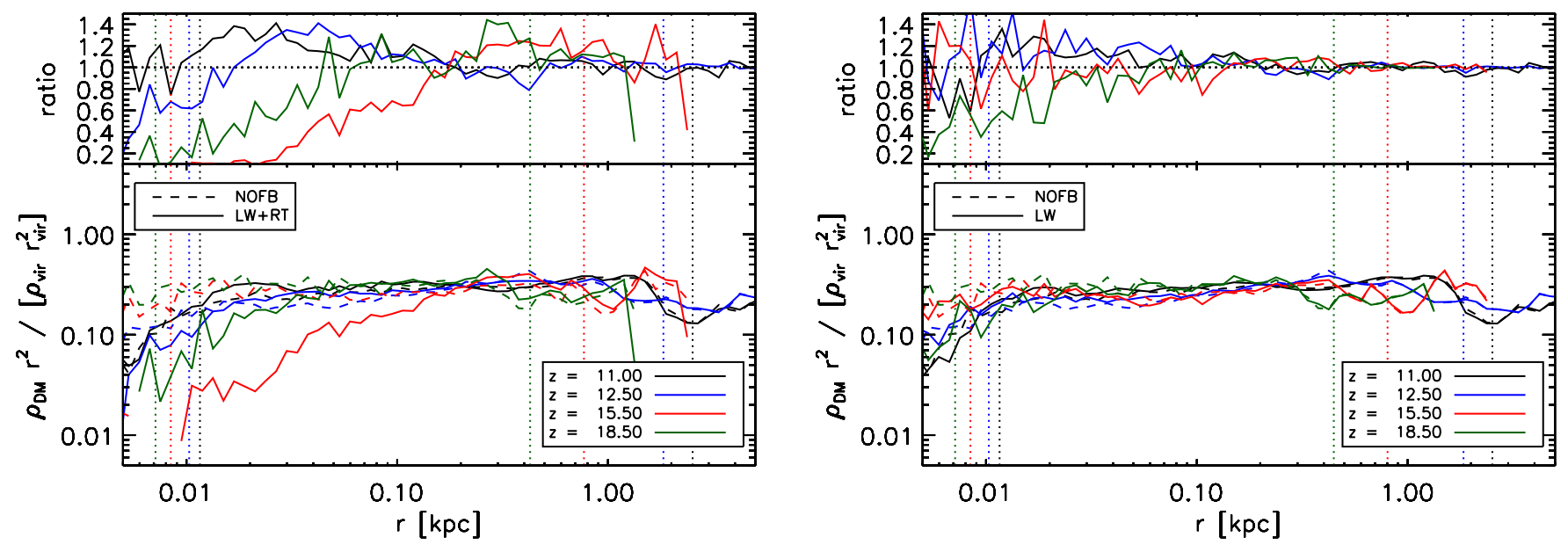

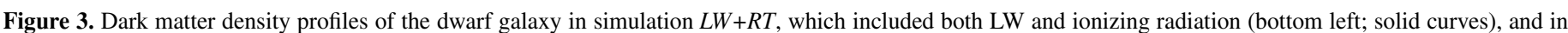

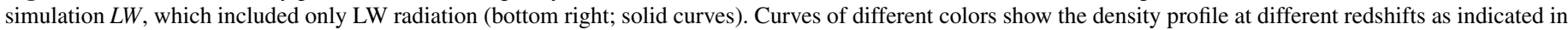

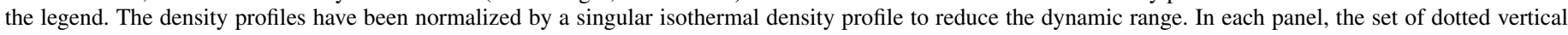

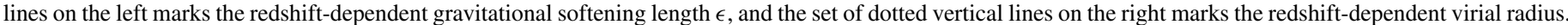

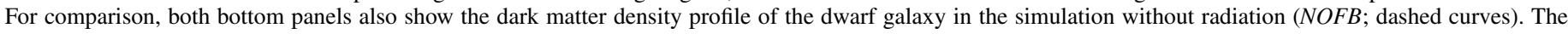

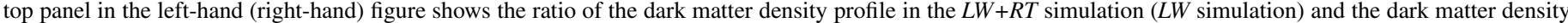

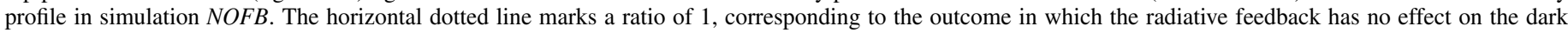

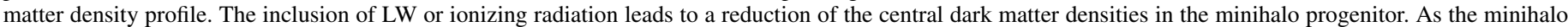

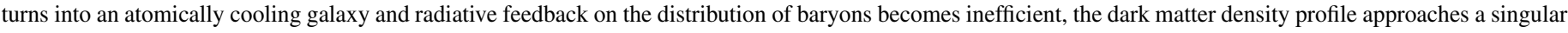
isothermal profile in all simulations.

(A color version of this figure is available in the online journal.)

models (Blumenthal et al. 1986; Gnedin et al. 2004, 2011). One may then expect the reverse of this process, i.e., the removal of gas from the halo center, to lead to a reduction in the concentration of the dark matter. Indeed, previous works have demonstrated the ability of SN explosions to lower the central dark matter densities in dwarf galaxies with masses $\gtrsim 10^{9} M_{\odot}$ at $z \lesssim 10$ (e.g., Navarro et al. 1996; Mashchenko et al. 2006, 2008; Governato et al. 2012).

Here we have shown that radiative feedback can have a qualitatively similar effect on the dark matter distribution in high-redshift minihalos. The reduction in the central dark matter densities due to radiative feedback is similar to that seen in the adaptive mesh refinement simulations of Wise \& Abel (2008b). In our simulation $L W+R T$, the centrally suppressed dark matter profiles shown in the left panel of Figure 3 can be approximated by a Navarro et al. (1997) profile and concentration parameter $\sim 2$ at $z=18.5$ and 15.5. The effect of radiative feedback on the dark matter profiles of high-redshift low-mass halos was also investigated by Ricotti (2003). The shape of dark matter profiles in a cosmological simulation including gas dynamics and radiative feedback was found to be similar, on average, to the shape in a simulation that was identical except that it only treated the dynamics of the dark matter. Our finding that radiative feedback can counteract the effects of gas condensation on the dark matter density profile is consistent with the results in Ricotti (2003) and Wise \& Abel (2008b). However, Ricotti (2003) also points out that there are significant statistical variations in the shape of the dark matter profile of halos at fixed mass and redshift in the simulation that included gas physics. We therefore caution against overinterpreting our conclusions drawn from the investigation of a single dwarf halo.

\subsection{The Disks}

Figure 4 shows a sequence of snapshots of the gas density distribution centered on the dwarf galaxy in simulation $L W+R T$. At the final simulation redshift, the gas at the halo center is organized in a massive central, spherical clump, an inner compact disk, and an outer extended disk. The panels can be compared with Figure 8 of Pawlik et al. (2011), which shows the gas densities in our earlier simulation identical to simulation $L W+R T$ discussed here except that it did not include star formation or radiation. The comparison shows that the radiative feedback is weak and the inclusion of star formation and LW and ionizing radiation does not prevent the formation of the disks seen in that earlier simulation.

The sequence of events leading to the formation of the two disks is very similar with and without the inclusion of radiation, and the reader may therefore refer to Pawlik et al. (2011) for additional details. A major merger at redshift $z \lesssim 15$ channels gas in the halo center. This leads to the formation of the first gaseous disk by $z=13.5$. The disk subsequently develops spiral arms. The spiral arms exert torques that imply an outward transport of angular momentum in the disk gas. As a consequence, the disk shrinks in size. At $z \approx 12$, a sequence of minor mergers replenishes the halo center with gas, producing the second gaseous disk. This disk remains spatially extended until the end of the simulation. The second disk surrounds the first disk, and the two have orientations tilted with respect to each other. This tilt is an interesting consequence of the hierarchical assembly of the emerging dwarf galaxy (e.g., Roškar et al. 2010; Pawlik et al. 2011). The formation redshifts of the disks are similar in all simulations and hence insensitive to the inclusion of radiation.

Photoheating creates low-density regions in the disk gas, leaving behind a disk morphology more complex than in the absence of photoheating (compare, e.g., the bottom right panel of Figure 4 with the corresponding panel in Figure 8 of Pawlik et al. 2011). These regions remain, however, locally confined, and the disks remain, as a whole, intact and relatively unaffected by photoheating. This insensitivity of the gas to photoheating is consistent with the fact that at the time of formation of the disks, i.e., at $z \lesssim 15$, the galaxy halo has already entered the atomic cooling regime. Hence, its gravitational potential is sufficiently 

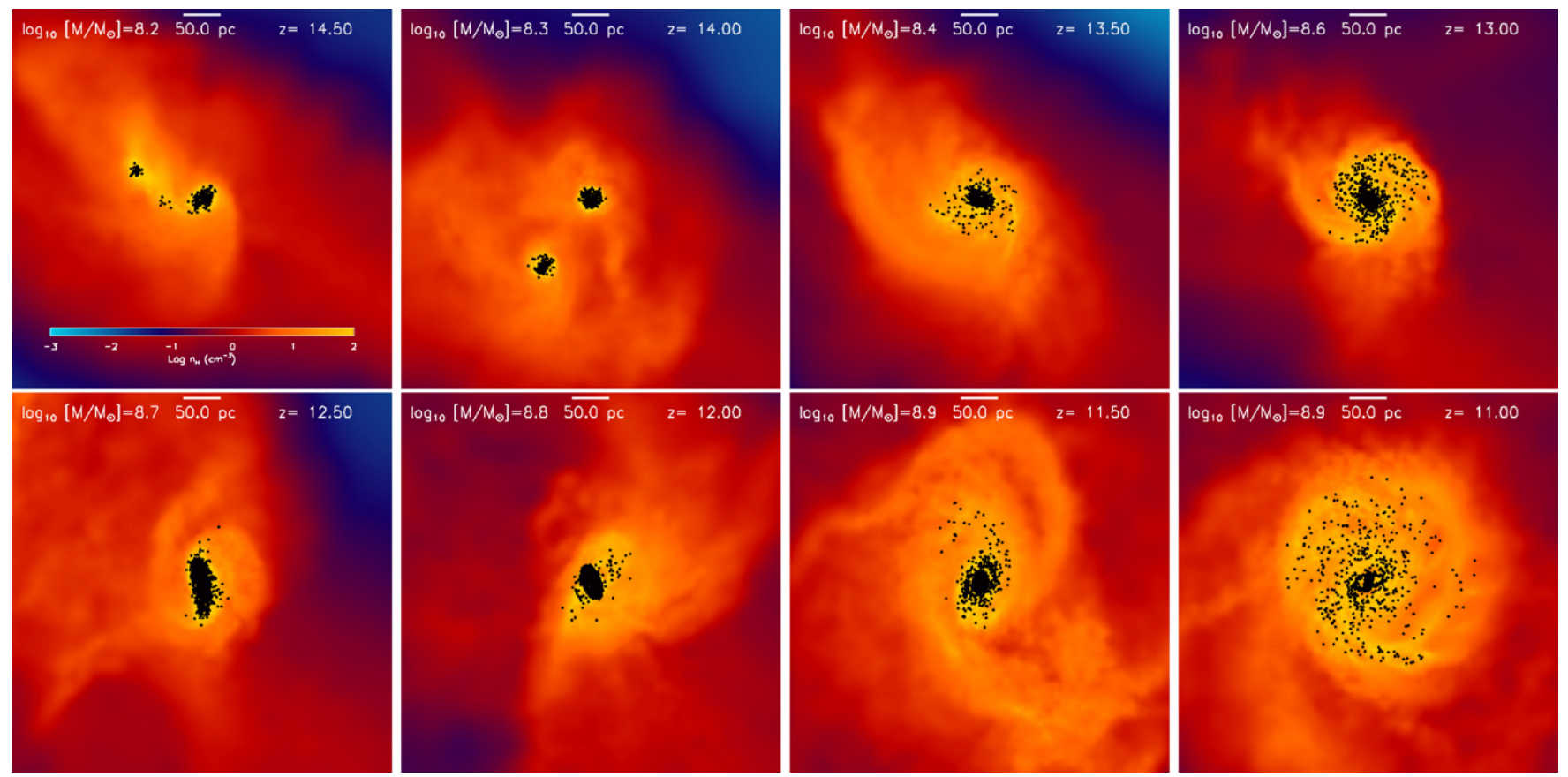

Figure 4. Assembly history of the disks in simulation $L W+R T$ which includes both $L W$ and ionizing radiation. The panels present face-on views of the assembling dwarf galaxy and show the evolution of gas densities in the redshift range $11.0 \leqslant z \leqslant 14.5$ in cubical slices of linear size $0.5 \mathrm{kpc}$. The locations of star particles emitting LW and ionizing radiation are indicated with black dots. This set of panels can be directly compared with that in Figure 8 of Pawlik et al. (2011), which shows the gas densities in a simulation identical to simulation $L W+R T$ presented here except that it did not include star formation or radiation. Radiative feedback creates locally confined low-density cavities (best visible in the bottom right panel), but it is not sufficiently strong to prevent the assembly of the disks inside the atomically cooling dwarf galaxy.

(A color version of this figure is available in the online journal.)
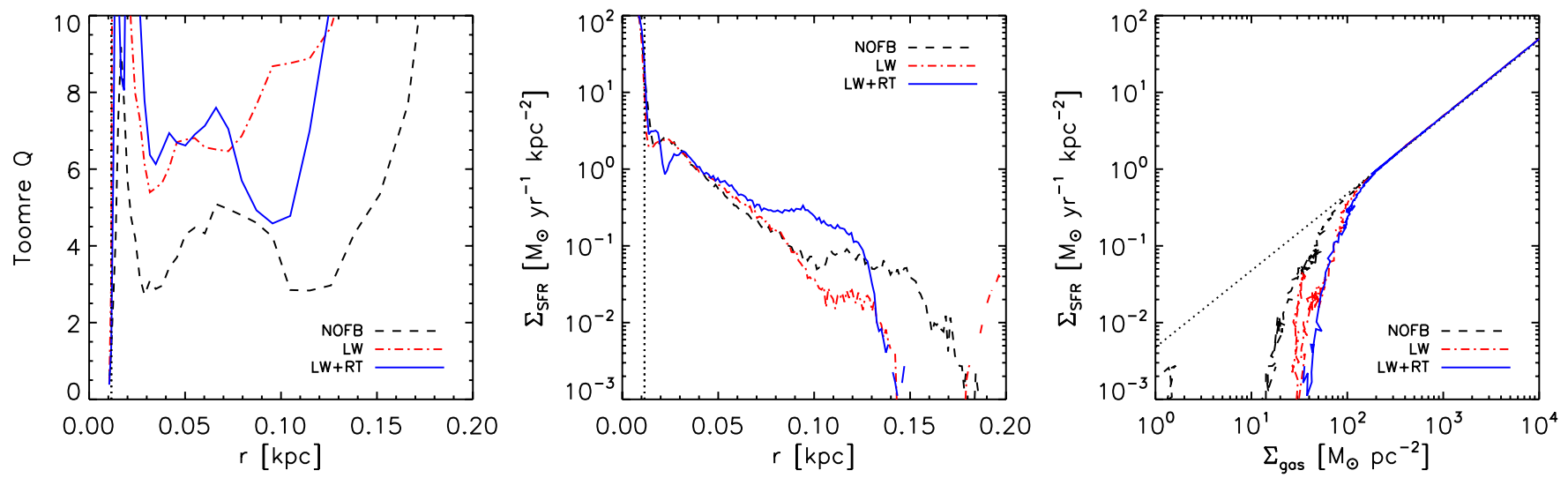

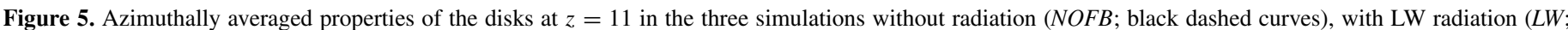

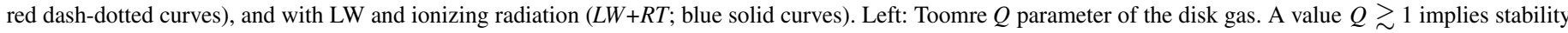

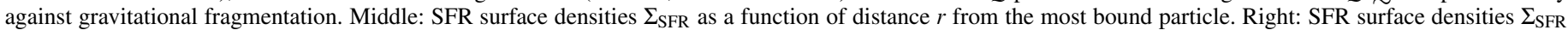

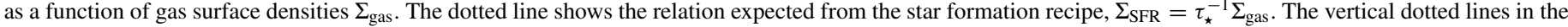
left and middle panels mark the softening scale $\epsilon$

(A color version of this figure is available in the online journal.)

deep to confine photoheated gas in its interior. A positive feedback loop which consists of high gas densities confining the photoheated $\mathrm{H}$ II regions allows the gas to collapse to increasingly higher densities, further confining the photoheated gas.

The left panel of Figure 5 shows the Toomre parameter, averaged in annular bins, of the disk gas at the final simulation redshift, $Q=c_{\mathrm{s}} \kappa /(\pi G \Sigma)$, where $c_{\mathrm{s}}$ is the adiabatic sound speed, $\kappa=\left(4 \Omega^{2}+r d \Omega^{2} / d r\right)^{1 / 2}$ is the epicyclic frequency, and $\Omega$ is the angular velocity of the disk gas (Toomre 1964). In all three simulations $Q \gtrsim 1$ at radii larger than the gravitational softening length, implying that the disk configuration is stable against fragmentation. Note that the inclusion of LW and ionizing radiation increases disk stability by increasing the sound speed. The inclusion of ionizing radiation further helps to preserve the disks because photoheating evaporates the gas from the lowmass halos merging with the dwarf galaxy. In the absence of feedback, on the other hand, baryon-rich low-mass halos that pass through the disks can disturb the disks significantly (e.g., Figure 3 in Pawlik et al. 2011).

The middle and right panels of Figure 5 show spherically averaged profiles of the SFR surface densities (middle), and the Kennicutt-Schmidt relation (right), i.e., the relation between SFR surface density and gas surface density, at the final simulation redshift. The Kennicutt-Schmidt relation is characterized by a power-law behavior and a suppression of star formation 

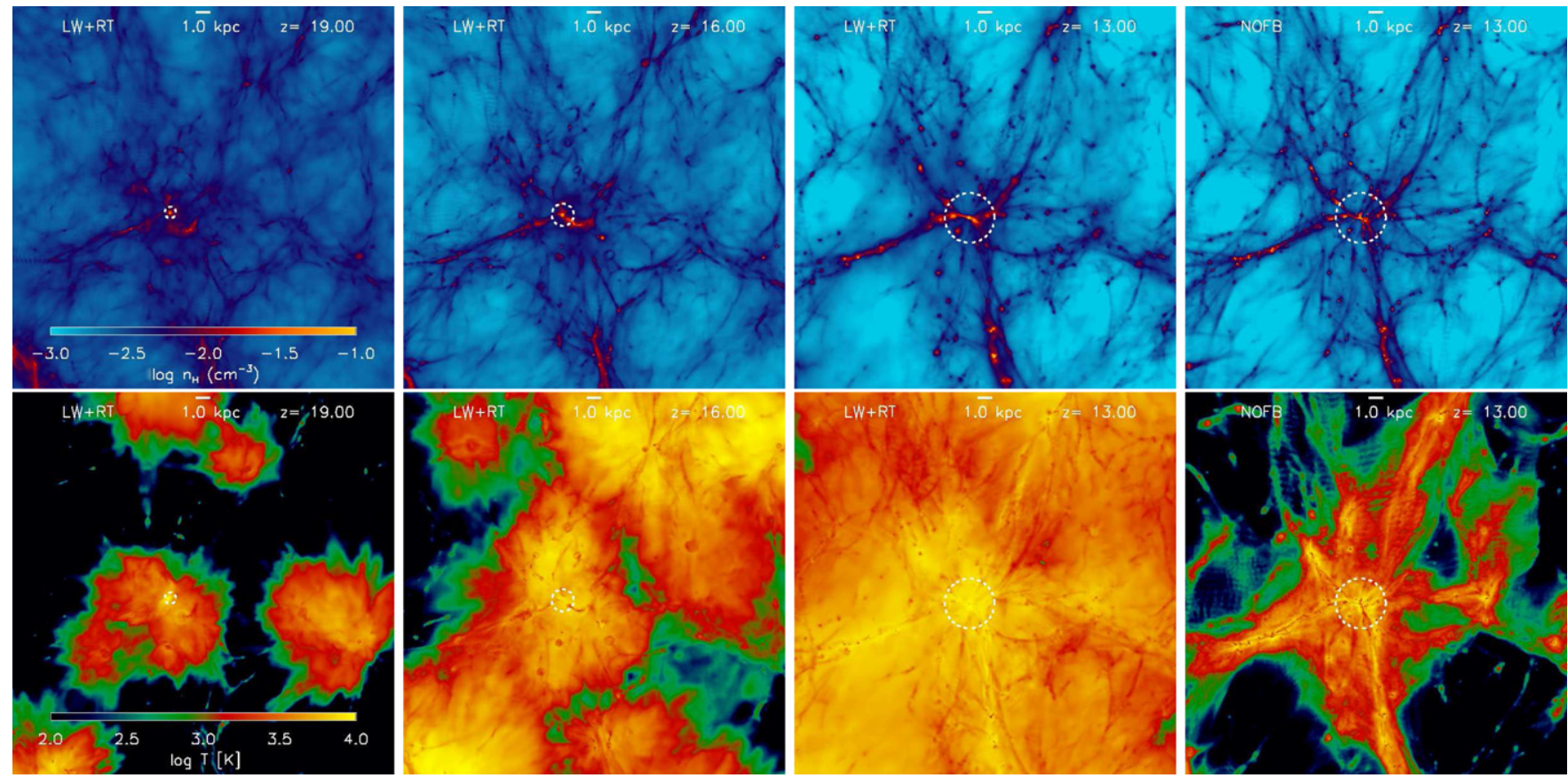

Figure 6. Evolution of gas densities (top) and temperatures (bottom) in simulation $L W+R T$ at three representative redshifts $z=19,16$, and 13 (from left to right). For comparison, the rightmost panels show the gas densities and temperatures in simulation NOFB at $z=13$. The cubical slices of fixed physical dimensions are centered on the comoving position of the most bound particle in the dwarf galaxy at $z=10$. The dashed circles are centered on the dwarf galaxy progenitor and have a radius equal to the virial radius of the progenitor. Photoheating raises the gas temperature to $\sim 10^{4} \mathrm{~K}$. The implied increase in gas pressure evaporates gas from inside low-mass halos and smooths gas density fluctuations in the IGM.

(A color version of this figure is available in the online journal.)

below gas surface densities $\lesssim 10^{2} M_{\odot} \mathrm{pc}^{-2}$. The suppression is a result of the star formation recipe, which limits star formation to densities above $500 \mathrm{~cm}^{-3}$. At the final simulation redshift, such densities are realized both in the central region and in the disks, and the galaxy shows a spatially extended morphology of star formation. In contrast, star formation in the galaxy before disk formation is limited to the central region (see Figure 4). The Kennicutt-Schmidt power-law behavior can be understood by writing $\Sigma_{\mathrm{SFR}}=\tau_{\text {gas }}^{-1} \Sigma_{\text {gas }}$, where $\tau_{\text {gas }}=\rho / \dot{\rho}_{\star}$ is the gas consumption time. Using $\tau_{\text {gas }}=\tau_{\star}$ (Equation (1)), we find $\Sigma_{\mathrm{SFR}}=\tau_{\star}^{-1} \Sigma_{\text {gas }}$. This relation is shown with the dotted line in Figure 5, and the results from the simulation are in close agreement with it.

Taken at face value, the large Toomre $Q$ values that indicate stability against disk fragmentation seem inconsistent with the non-zero SFRs of the disks. However, our simulations do not have sufficient resolution or the physical detail required to study fragmentation of the disks into individual stars. We have therefore employed a phenomenological model for star formation according to which stars form from gas with densities larger than the adopted threshold density for star formation, an approach employed in most galaxy formation simulations. It remains open if, at higher resolution or greater physical detail, the disks in our simulation would fragment to form stars, or if they would remain stable, possibly feeding a central massive black hole (e.g., Eisenstein \& Loeb 1995; Koushiappas et al. 2004; Lodato \& Natarajan 2006). Addressing these issues in cosmological simulations such as the simulations here is computationally challenging. Simulations of isolated disk galaxies have shown that star formation is slower in disks that are more stable as quantified by the smallest Toomre $Q$ value in the disk (Li et al. 2005). It may therefore be that star formation in high-redshift low-mass disk galaxies is less efficient than implied by our simulations.

\section{REIONIZATION AND RADIATIVE FEEDBACK FROM THE FIRST STARS}

Figure 6 shows projections of the gas density (top) and temperature (bottom) in cubical slices through the refinement region in simulation $L W+R T$ at three representative redshifts $z=19,16$, and 13 (from left to right). For comparison, the figure also shows the gas density and temperature at $z=13$ in the corresponding slice through simulation NOFB (rightmost panels). In the following, we discuss the impact of LW and ionizing radiation on the properties of the IGM and the formation of low-mass galaxies in the neighborhood of the simulated dwarf galaxy.

The feedback processes exerted by radiation are well known and are briefly summarized here. LW radiation photodissociates molecular hydrogen, reducing the ability of low-mass halos to condense their gas. This suppresses star formation, providing a negative feedback (e.g., Haiman et al. 1997). Ionizing radiation photoheats the IGM to $\sim 10^{4} \mathrm{~K}$, and the implied Jeans filtering impedes the accretion of gas into halos with virial temperatures $\lesssim 10^{4} \mathrm{~K}$ (Shapiro et al. 1994; Gnedin \& Hui 1998). In addition, photoheating evaporates gas from low-mass halos and reduces the ability of gas to cool inside them (e.g., Efstathiou 1992; Thoul \& Weinberg 1996; Wiersma et al. 2009). On the other hand, photoionization generates free electrons, which catalyze the formation of molecular hydrogen, thus increasing the ability of gas inside low-mass halos to cool and form stars (e.g., Ricotti et al. 2002; Oh \& Haiman 2002). Photoionization therefore provides both a negative and a positive feedback on star 

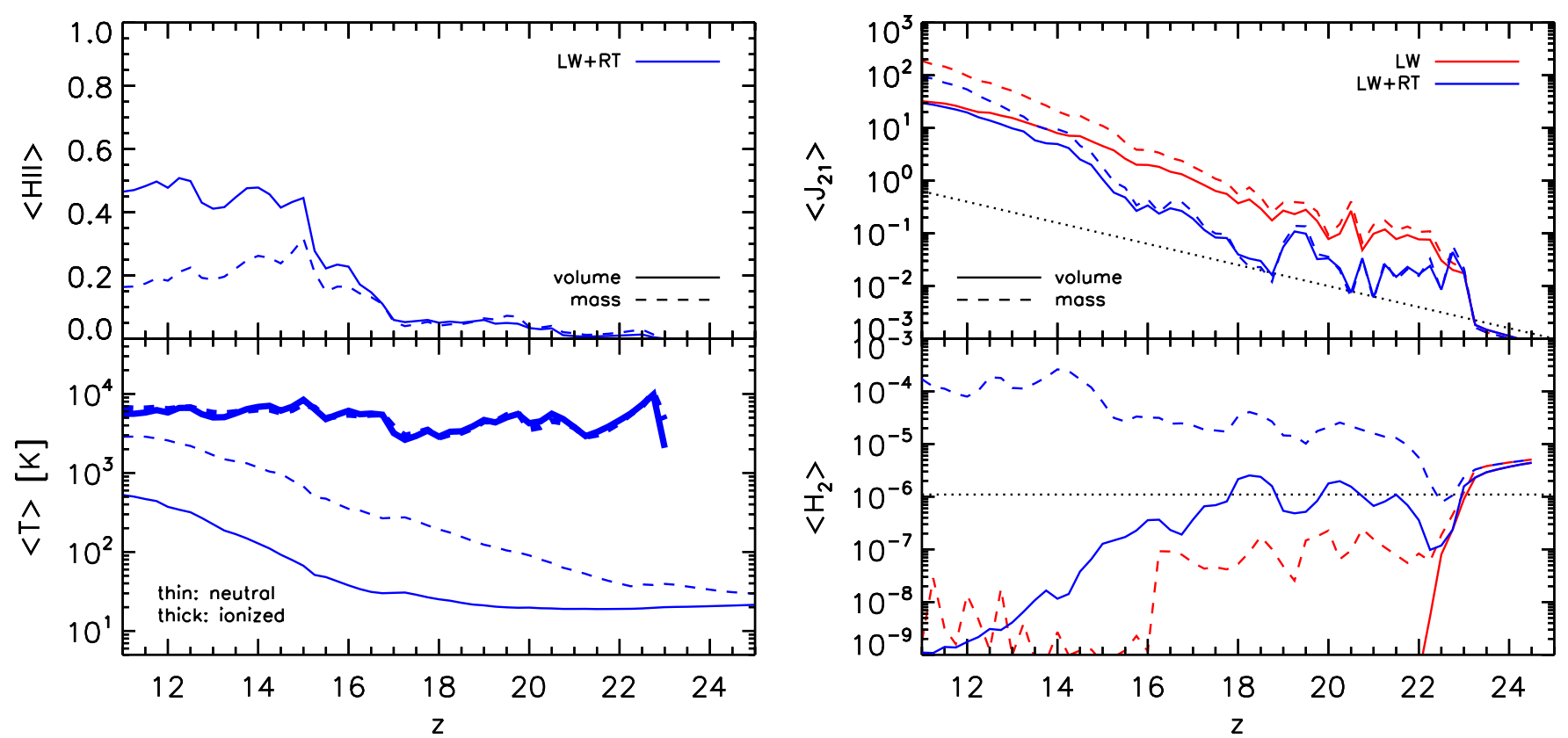

Figure 7. Reionization history of the immediate neighborhood of the dwarf galaxy, defined by the sphere of comoving radius $\approx 100 \mathrm{kpc}$ centered on the comoving position of the dwarf galaxy at $z=10$, the same position used to center the images in Figure 6. In all panels, solid curves show volume-weighted averages, and dashed curves show mass-weighted averages. Top left: mean ionized fractions $\left\langle\eta_{\mathrm{H} \mathrm{II}}\right\rangle$. Bottom left: mean gas temperatures $\langle T\rangle$. Thick (thin) lines show average temperatures including only gas that is ionized (neutral), i.e., gas with $\eta_{\mathrm{H} \text { I }}>10^{-3}\left(\eta_{\mathrm{H} \text { II }} \leqslant 10^{-3}\right)$. Top right: mean normalized LW intensities $\left\langle J_{21}\right\rangle$. The average was computed in $\log$-space to reduce the dynamic range, i.e., $\left\langle J_{21}\right\rangle \equiv 10^{\left\langle\log _{10} J_{21}\right\rangle}$. The dotted line shows the intensity of the imposed LW background. Bottom right: mean molecular hydrogen fractions $\left\langle\eta_{\mathrm{H}_{2}}\right\rangle$. The dotted horizontal line marks the initial molecular hydrogen fraction, $\eta_{\mathrm{H}_{2}}=1.1 \times 10^{-6}$. The dwarf galaxy highly but not fully reionizes the gas in its overdense neighborhood by the final simulation redshift, $z=11$.

(A color version of this figure is available in the online journal.)

formation. A comprehensive overview of the effects of radiative feedback can be found in, e.g., Ciardi \& Ferrara (2005).

\subsection{The Start of Reionization}

Figure 7 quantifies the ionization and thermal history (left) as well as the evolution of LW intensities and of the molecular hydrogen fraction (right) around the dwarf galaxy in simulation $L W+R T$ (blue curves). The neighborhood considered here is defined, for simplicity of geometry and to reduce boundary artifacts, as the sphere of comoving radius $5 r_{\text {vir,com }}(z=10)$, where $r_{\mathrm{vir}, \mathrm{com}}(z=10) \approx 34 \mathrm{kpc}$ comoving is the virial radius of the dwarf galaxy at $z=10$. We center the sphere on the comoving position of the most bound particle of this galaxy at $z=10$, and this is the same position on which the slices in Figure 6 are centered. The spherical neighborhood is contained in the refinement region at all redshifts. Our qualitative discussion below is not sensitive to the precise definition of the neighborhood adopted here. Volume-weighted (mass-weighted) averages are obtained by weighting with the SPH kernel volume (particle mass).

The fraction of the volume ionized (solid) increases with decreasing redshift until $z \lesssim 15$, after which it remains approximately constant at $\left\langle\eta_{\mathrm{H} \text { II }}\right\rangle_{V} \approx 0.5$. The fraction of the mass ionized (dashed) shows a qualitatively similar behavior, but it slightly decreases after $z \lesssim 15$ and reaches $\left\langle\eta_{\mathrm{H} \text { II }}\right\rangle_{m} \approx 0.2$ at the final simulation redshift. The redshift below which the ionized fractions no longer increase is similar to the redshift at which the galaxy evolves into an atomically cooling object. The fraction of the mass ionized is initially close to but slightly larger than the fraction of the volume ionized, but this changes at $z \lesssim 19$ after which the fraction of ionized mass becomes increasingly lower than the fraction of the ionized volume. Reionization thus proceeds from the inside-out, from the dense halo gas into the diffuse IGM, as expected (e.g., Wise \& Abel 2008a). The mass- and volume-weighted average gas temperature of the ionized gas, which we have defined here as gas with ionized hydrogen fraction $\eta_{\mathrm{H} \text { II }}>10^{-3}$, fluctuates between 3000 and 10,000 K, in good agreement with the results in Figures 12 and 13 in Wise \& Abel (2008a).

Figure 7 shows that the ionization of the region around the main galaxy in simulation $L W+R T$ is accompanied by an increase in the average LW intensities. Before the formation of the first star, the average LW intensity is close to but slightly smaller than the intensity of the imposed LW background (dotted line), a consequence of self-shielding. Emission of stellar radiation increases the LW intensity above the background, and the latter quickly becomes unimportant. At $z \lesssim 16$ the LW intensities increase more rapidly and approach the intensities found in simulation $L W$ that only included LW radiation (red curves), and the LW radiation efficiently destroys the molecular hydrogen. However, the mass-weighted fraction remains significantly larger than the volume-weighted fraction, mostly because of self-shielding. The comparison with simulation $L W$ shows that the inclusion of ionizing radiation promotes the formation of molecular hydrogen (e.g., Haiman et al. 1996a; Ricotti et al. 2001; Oh \& Haiman 2002).

The dwarf galaxy highly but not fully ionizes its neighborhood by $z=11$. This is likely due to a combination of reasons. First, as the galaxy evolves into an atomically cooling object, photoheating is no longer able to substantially reduce the gas densities inside it (see Section 2). Ionizing photons emitted by the stellar sources are then efficiently consumed by recombinations in the dense gas, thus limiting the ionizing impact on the 
surrounding IGM. Second, as we will discuss in Section 5.2, star formation in the dwarf galaxy and its neighboring galaxies exerts a strong negative feedback on neighboring low-mass galaxies, suppressing star formation inside them. These galaxies thus cannot significantly contribute to reionizing the gas. Finally, the considered volume lacks more massive galaxies, which would be robust against the feedback from the dwarf galaxy and could potentially help reionizing the gas. This is an artifact of the refinement region being too small to contain these rarer halos. Note also that the galaxy resides in a highly overdense region, and our computation of the mean ionized fraction includes contributions from the self-shielded neutral gas in halos inside this region.

\subsection{Feedback on Galaxy Formation}

Figure 8 shows the evolution of the minimum virial mass of star-forming halos in the refinement region, as found in simulation $L W+R T$ in which stars emitted both $L W$ and ionizing radiation $(L W+R T$; blue). The corresponding evolutions in the simulation in which stars emitted LW but not ionizing radiation $(L W$; red), and in the simulation in which no radiation was present (NOFB; black), are also shown. We computed the minimum mass both by considering halos that form stars for the first time (crosses), which is hereafter referred to as the minimum collapse mass, and by considering all halos, including those that have previously formed stars (boxes with matching but lighter colors). Our definition of the minimum collapse mass is insensitive to our choice of the threshold density for star formation $n_{\mathrm{H}, \mathrm{SF}}=500 \mathrm{~cm}^{-3}$, at least in the absence of feedback from star formation, because the transition from densities $n_{\mathrm{H}} \gtrsim 10 \mathrm{~cm}^{-3}$ to $n_{\mathrm{H}} \lesssim 10^{3} \mathrm{~cm}^{-3}$ which bracket the adopted star formation threshold density then occurs within a narrow range of halo masses (see the bottom left panel in Figure 9).

In the absence of LW or ionizing radiation, the minimum collapse mass is consistent with the mass of halos at virial temperature $T_{\text {vir }} \approx 2200 \mathrm{~K}$ (Equation (6) with $\mu=1.22$ ) independent of redshift. This mass is consistent with but slightly larger than the masses $\sim 5 \times 10^{5}-10^{6} M_{\odot}$ at the time of gas collapse in previous simulations of minihalos (e.g., Yoshida et al. 2003; O'shea \& Norman 2007; Wise \& Abel 2008a; Greif et al. 2008). The small difference is probably a numerical artifact of our limited resolution, which is lower than those realized in the previous works. Differences are also expected because the mass of the halo that forms the first star is subject to statistical uncertainties related to the low number of investigated halos (e.g., Greif et al. 2008; O'shea \& Norman 2008), and because of differences in the strength of dynamical heating from tidal interactions and mass accretion (Yoshida et al. 2003; O'shea \& Norman 2007). The inclusion of LW radiation increases the minimum collapse mass. This increase in the minimum collapse mass due to photodissociation by LW radiation has been investigated in detail in a number of previous works (e.g., Machacek et al. 2001; Mesinger et al. 2006; O'shea \& Norman 2008; Safranek-Shrader et al. 2012).

The inclusion of both LW and ionizing radiation increases the minimum collapse mass in a similar manner but often by a smaller factor, demonstrating a positive feedback from the enhanced formation of molecular hydrogen in ionization fronts and fossil $\mathrm{H}$ II regions. Eventually, however, the negative feedback from photoheating outweighs the positive feedback, and there is no new star-forming halo inside the refinement region below $z \lesssim 13$. Star formation can still proceed in halos

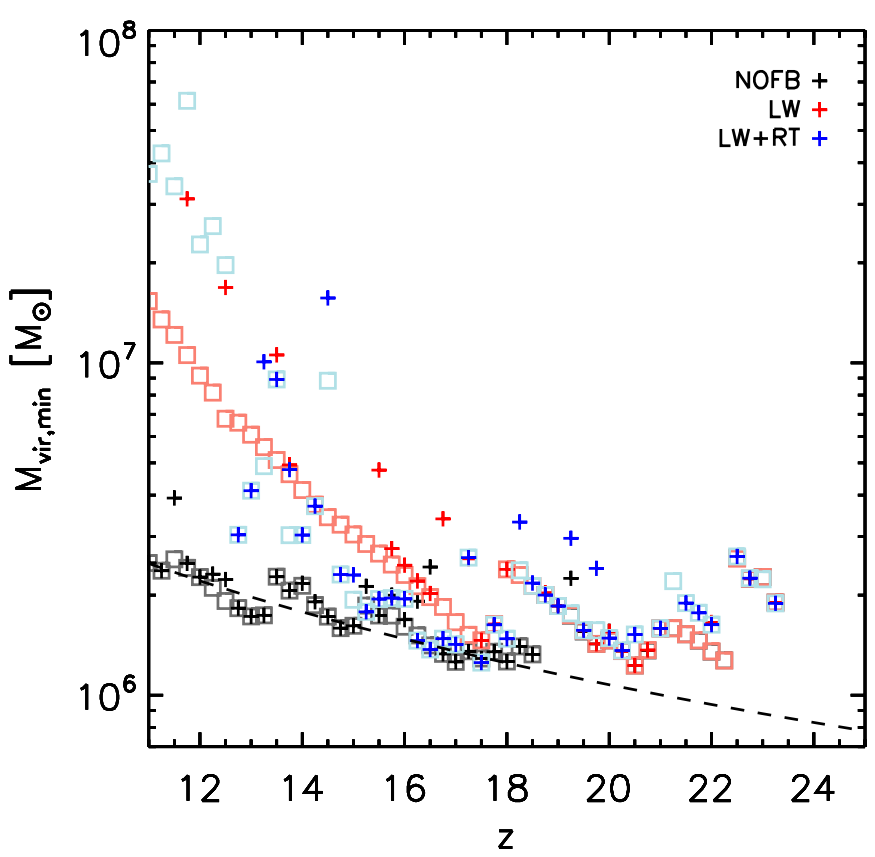

Figure 8. Minimum collapse mass, i.e., the mass of the lowest mass halo inside the refinement region that forms stars for the first time (crosses). The minimum collapse mass is shown both as inferred from simulation $L W+R T$ (blue), which included both dissociating and ionizing radiation, and, for comparison, in the simulations without radiation (NOFB; black), and dissociating radiation only ( $L W$; red). We also show the virial mass of the lowest mass halo that forms stars, including halos that have previously hosted star formation, in the same three simulations (squares with matching but lighter colors). The dashed curve shows a virial mass corresponding to a virial temperature $T_{\text {vir }}=2200 \mathrm{~K}$ (Equation (6) with $\mu=1.22$ ), which provides a good description of the evolution of the collapse mass in simulation $N O F B$. The minimum collapse mass is raised in simulation $L W$ due to photodissociation of molecular hydrogen by LW photons and in simulation $R T+L W$ additionally due to the Jeans filtering of the IGM. In the latter simulation, the enhanced molecular hydrogen fraction in fossil $\mathrm{H} \mathrm{II}$ regions can lead to a reduction in the minimum collapse mass below that in simulation $L W$.

(A color version of this figure is available in the online journal.)

that have previously formed stars, but the masses of these halos are significantly larger than those in simulation $N O F B$. This is the result of the Jeans filtering of the IGM, which impedes the accretion of gas on low-mass halos. The increased scatter in the minimum collapse mass in the presence of photoionization is an expression of the local nature of feedback from photoheating, which is limited to inside the H II regions.

The top panels of Figure 9 show the specific SFRs, i.e., the SFRs divided by virial mass, both as a function of virial mass (left) and of stellar mass (middle), and the stellar mass fractions (right) for the halos inside the high-resolution region at the final simulation redshift, $z=11$. The bottom panels of Figure 9 show the maximum gas densities inside the virial radius (left) and the baryon mass fractions as function of virial (middle) and stellar mass (right) for these halos.

In the simulation without radiation $(N O F B)$, the specific SFR $\sim 2 \times 10^{-10} \mathrm{yr}^{-1}$ is nearly independent of halo mass in the range $\sim 10^{7}-10^{9} M_{\odot}$. The specific SFR is reduced in a fraction of the halos with masses $\lesssim 10^{7} M_{\odot}$ just above the minimum collapse mass, which we attribute primarily to the effects of dynamical heating during the gravitational collapse of these halos (e.g., Yoshida et al. 2003). Indeed, the fact that the transition to high central gas densities occurs within a finite range of halo masses shows that dynamical heating plays a non-negligible role, and in the lowest mass halos this may be 

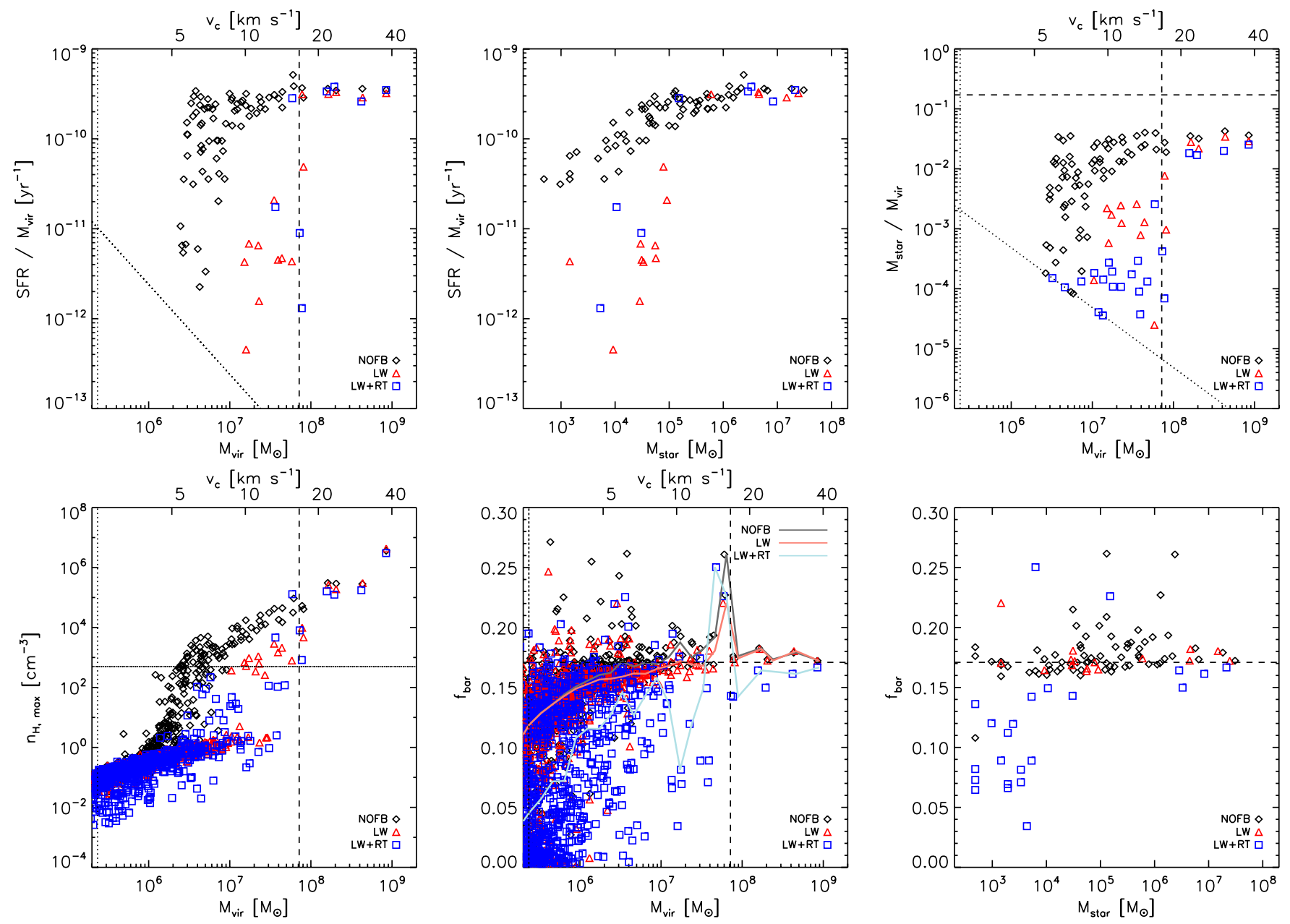

Figure 9. Properties of halos at $z=11$, the final simulation redshift, in the refinement region in the three simulations without radiation (NOFB; black diamonds), with only $L W$ radiation ( $L W$; red triangles), and with $L W$ and ionizing radiation $(L W+R T$; blue squares). Each symbol represents a single FOF halo. Dashed and dotted vertical lines, if shown, mark the virial mass of a halo with virial temperature $T_{\mathrm{vir}}=10^{4} \mathrm{~K}$ (Equation (6) with $\mu=0.6$ ), and the mass $100 \mathrm{~m}_{\mathrm{DM}}$. A horizontal dashed line, if shown, marks the universal baryon fraction. The top axis, if shown, labels the virial velocity $v_{\mathrm{c}}=\left(G M_{\mathrm{vir}} / r_{\mathrm{vir}}\right)^{1 / 2}=17.0\left(M / 10^{8} M_{\odot}\right)^{1 / 3}[(1+z) / 10]^{1 / 2} \mathrm{~km} \mathrm{~s}^{-1}$ (e.g., Equation (3.11) in Loeb 2010). Top left: SFR, normalized by virial mass, as a function of virial mass. Middle left: SFR, normalized by virial mass, as a function of stellar mass. Top right: stellar mass fraction. The diagonal dotted line marks the stellar mass fraction implied by the presence of a single star particle. Bottom left: maximum gas density. The horizontal line marks the star formation threshold density. Bottom middle: baryon fraction as a function of virial mass. The curves of matching but lighter colors show medians to help guide the eye. Bottom right: baryon fraction as a function of stellar mass. All quantities have been computed considering only matter inside the virial radius $r_{\text {vir }}$. Photodissociation of molecular hydrogen by LW radiation suppresses star formation completely only in minihalos with masses significantly below the atomic cooling limit. Photoheating, on the other hand, suppresses star formation also in more massive minihalos. The baryon fraction in simulation $L W+R T$ is lowest in non-star-forming halos with masses $\lesssim 10^{6} M_{\odot}$, in which it is strongly reduced by radiative feedback from external sources, and in star-forming halos with masses $10^{7}-10^{8} M_{\odot}$, in which it is additionally reduced by radiative feedback from internal sources.

(A color version of this figure is available in the online journal.)

amplified by the limited mass resolution we afford. Note that some of the halos show an increased baryon fraction $f_{\text {bar }} \gtrsim 0.2$ as a result of dynamical interaction and ongoing mergers with other halos.

The inclusion of LW radiation implies a complete suppression of star formation only in halos with masses $\lesssim 2 \times 10^{7} M_{\odot}$, corresponding to virial temperatures significantly below $10^{4} \mathrm{~K}$ (vertical dashed line). This is in qualitative agreement with the results from previous high-resolution simulations of the collapse of minihalos in the presence of an LW radiation background. These works demonstrated that as the minihalo mass approaches the atomic cooling limit, the presence of LW radiation cannot prevent the buildup of molecular hydrogen, because it is catalyzed by the elevated electron fraction inside central structure formation shocks (e.g., Wise \& Abel 2007a; O'shea \& Norman 2008), and also because of self-shielding (e.g., Ahn \& Shapiro 2007; Susa 2007). In contrast, the additional inclusion of ioniz- ing radiation can potentially suppress star formation in all halos below the atomic cooling limit by evaporating the gas from the halo centers. However, as we have already discussed above in Figure 8 , inspection of the stellar mass fractions reveals that in simulation $L W+R T$, star formation is possible down to lower halo masses than in simulation $L W$, a consequence of the positive feedback from ionization ahead of ionization fronts and recombinations of $\mathrm{H}$ II regions on the formation of molecular hydrogen (Ricotti et al. 2002, 2008).

The simultaneous inclusion of both LW and ionizing radiation reduces the average baryon fractions in nearly the full range of simulated halo masses. The reduction of the baryon fraction is strongest, on average, for the lowest-mass halos with masses $\lesssim 10^{6} M_{\odot}$, and for halos in the intermediate-mass regime, with masses in the range $10^{7}-10^{8} M_{\odot}$. Because halo masses $\lesssim 10^{6} M_{\odot}$ are below the minimum collapse mass, halos in this mass range do not form stars, and hence their baryon 
fraction is reduced with respect to that in simulation $N O F B$ due to the effects of radiation from external sources, and due to Jeans filtering in the photoheated IGM. More massive halos may accrete gas despite external feedback and Jeans filtering, which explains why the baryon fraction increases, on average, in the halo mass range $\sim 10^{6}-10^{7} M_{\odot}$. Halos in the range $10^{7}-10^{8} M_{\odot}$ are efficiently forming stars, and therefore their baryon fractions are strongly reduced by radiative feedback from internal sources. Finally, halos with masses $\gtrsim 10^{8} M_{\odot}$, corresponding to virial temperatures $\gtrsim 10^{4} \mathrm{~K}$, are robust not only against Jeans filtering and feedback from external sources, but also against the feedback from internal sources. The baryon fraction of these halos is reduced primarily because there was not yet enough time for accretion to compensate for the mass loss in past episodes of gaseous outflows.

Ricotti et al. (2008) investigated radiative feedback from the first galaxies using cosmological simulations of comoving size $1-2 h^{-1} \mathrm{Mpc}$ including processes such as chemical enrichment not treated here. Utilizing resolution similar to that realized here, they demonstrated that the positive feedback due to enhanced molecular hydrogen formation near ionization fronts and inside recombining $\mathrm{H}$ II regions can be very strong and compensate for the negative feedback due to photodissociation of molecular hydrogen (see also Ricotti et al. 2002). Our simulations may underestimate this positive feedback because we do not capture the spectral hardening of the ionizing radiation, implying less ionization to stimulate the formation of hydrogen ahead of ionization fronts. Ricotti et al. (2008) further demonstrated that the stellar mass fractions of the lowest mass halos are characterized by a large scatter at fixed halo mass. Our simulations do not find as large a scatter, which may be a consequence primarily of the larger star particle mass and statistical limitations caused by the small size of the refinement region in our simulations, although other differences between the simulations may contribute. We do however find a similar large scatter in the baryon fraction at low halo masses, and we also agree with Ricotti et al. (2008) that this scatter is driven by radiative feedback from both external and internal sources.

\section{PROSPECTS FOR OBSERVATIONS WITH JWST}

In this section, we estimate the fluxes of stellar and recombination radiation expected from the dwarf galaxy simulated here. We use these estimates to discuss the detectability of the first galaxies with the upcoming JWST (e.g., Gardner et al. 2006). We focus on the non-ionizing UV continuum at wavelengths around $1500 \AA$ (hereafter UV1500), and on the Ly $\alpha$ recombination line. We have presented initial estimates of the expected flux in Pawlik et al. (2011). The discussion here improves on our earlier work by basing the flux estimates on the dwarf galaxy simulation $L W+R T$ presented in the current work. This simulation, which tracked the emission of LW and ionizing radiation from massive metal-free stars, provides us with direct predictions of the SFRs in low-mass high-redshift galaxies evolving under the radiative feedback from the first stars.

JWST will image high-redshift galaxies in the UV1500 continuum using NIRCam. JWST will also perform spectroscopic observations of these galaxies in the Ly $\alpha$ line using NIRSpec. Observations of the UV continuum are routinely used to infer the SFR density in the high-redshift universe (e.g., Bunker et al. 2004; Sawicki \& Thompson 2006; Bouwens et al. 2009; Finkelstein et al. 2010), a key quantity which not only constrains the nature of the stellar populations (e.g., Dunlop et al. 2012; Bouwens et al. 2010), but also allows one to address the capa- bility of galaxies to reionize the universe (e.g., Stiavelli et al. 2004; Bouwens et al. 2009; Finkelstein et al. 2010). Observations of the Ly $\alpha$ line, on the other hand, have enabled, e.g., spectroscopic confirmation of galaxy candidates well into the epoch of reionization (e.g., Rhoads et al. 2004; Iye et al. 2006; Lehnert et al. 2010; Ono et al. 2012).

JWST will further enable spectroscopic observations of highredshift galaxies in the He1640 line, using NIRSpec, and in the $\mathrm{H} \alpha$ line, using MIRI. The intrinsic strength of the $\mathrm{H} \alpha$ line is weaker by a factor of about 10 than that of the Ly $\alpha$ line (e.g., Schaerer 2003). Unlike the Ly $\alpha$ line, the $\mathrm{H} \alpha$ line is, however, not affected by resonant scattering (e.g., Loeb \& Rybicki 1999; Santos 2004), rendering it a potentially competitive probe of high-redshift galaxy formation. The He1640 line, on the other hand, is highly sensitive to the metallicity and IMF of the stellar populations, and a high ratio of luminosities in the He1640 to $\mathrm{Ly} \alpha$ or $\mathrm{H} \alpha$ lines has been suggested as a smoking gun for the existence of massive metal-free stars (e.g., Tumlinson et al. 2001; Oh et al. 2001; Bromm et al. 2001). A detection of the He1640 line would therefore put strong constraints on current theories of metal enrichment and star formation in the high-redshift universe, but is extremely challenging because of its weak intrinsic strength (e.g., Zackrisson et al. 2011; Cai et al. 2011; Inoue 2011). Because we find that both the $\mathrm{H} \alpha$ and He1640 line fluxes expected from dwarf galaxies such as simulated here are generally too weak to be observed with JWST, we do not further discuss these emission lines. However, the reader may refer to our earlier discussion of the observability of the first galaxies in $\mathrm{H} \alpha$ and $\mathrm{He} 1640$ in Pawlik et al. (2011).

We use the Schaerer (2003) population synthesis models for constant star formation to convert the SFRs of the simulated dwarf galaxy in simulation $L W+R T$, which included both $L W$ and ionizing radiation, into intrinsic luminosities in the Ly $\alpha$ recombination line, and in the UV continuum. The Schaerer (2003) models require us to specify the IMF and the metallicities of the stellar populations, neither of which is predicted by our simulations and hence has to be assumed. To bracket plausible scenarios we repeat our analysis for three models (see the discussion in Pawlik et al. 2011). The first model assumes that stellar populations consist of metal-free very massive stars, which we describe by employing the Schaerer (2003) model with zero metallicity and an IMF with Salpeter slope in the stellar mass range 50-500 $M_{\odot}$ (hereafter top-heavy IMF). This is the same model employed to compute the ionizing and LW luminosities of the stellar bursts in our simulations. The second model assumes an IMF with Salpeter slope in the range 1-100 $M_{\odot}$ (hereafter normal IMF) and zero metallicity. The third and final model assumes the same IMF as the second model, but a non-zero (but low) metallicity $Z=5 \times 10^{-4} Z_{\odot}$.

We convert the line luminosities into observed line fluxes using $^{4} f\left(\lambda_{\mathrm{o}}\right)=L\left(\lambda_{\mathrm{e}}\right) /\left[4 \pi d_{\mathrm{L}}^{2}(z)\right]$, where $L\left(\lambda_{\mathrm{e}}\right)$ is the intrinsic Ly $\alpha$ line luminosity and $d_{\mathrm{L}}(z)$ is the luminosity distance to redshift $z$. The continuum luminosities are converted into observed flux densities using an analogous equation (Equation (5) in Pawlik et al. 2011). The line luminosities and the nebular contribution to the UV continuum luminosities are derived assuming that all ionizing photons are absorbed, i.e., the escape fraction is $f_{\text {esc }}=0$, thus maximizing the flux in the recombination lines. The line luminosities can be rescaled to the case of non-zero escape fractions by multiplication with $\left(1-f_{\mathrm{esc}}\right)$.

\footnotetext{
4 This differs from Pawlik et al. (2011), where we converted the line flux into an equivalent flux density (Equation (4) in that work).
} 

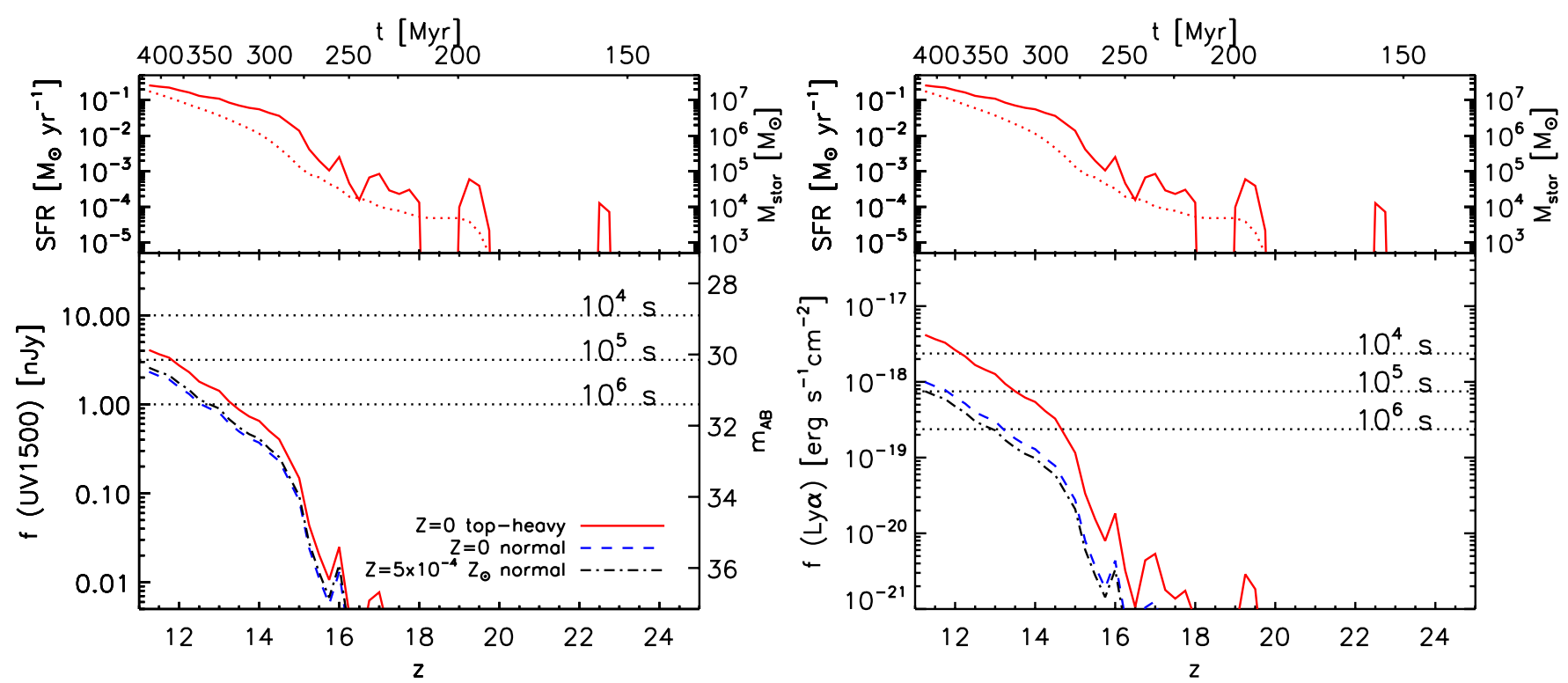

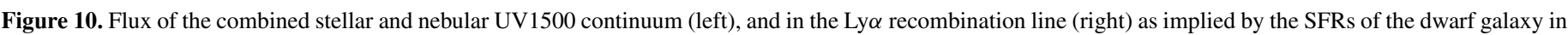

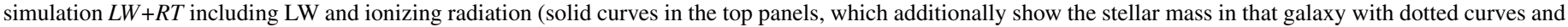

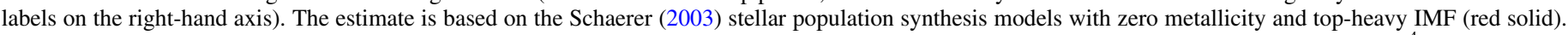

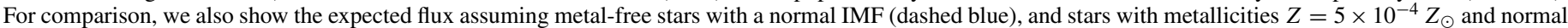

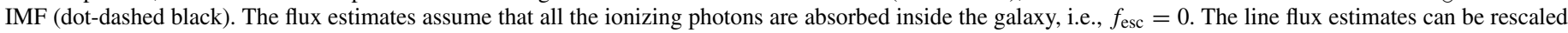

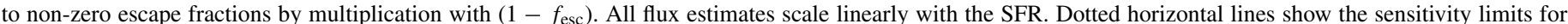

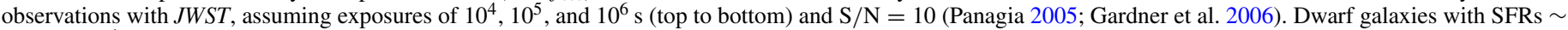
$0.1 M_{\odot} \mathrm{yr}^{-1}$ such as simulated here will be among the faintest galaxies JWST will detect in deep exposures of the $z \gtrsim 10$ universe.

(A color version of this figure is available in the online journal.)

We ignore the effects of resonant scattering on the observed Ly $\alpha$ line fluxes. Ly $\alpha$ RT simulations show that such effects can be very important, but they often depend sensitively on the specific structure of the investigated galaxies and hence are difficult to generalize (e.g., Verhamme et al. 2008; Dijkstra \& Kramer 2012). Finally, our flux estimates scale linearly with the SFRs.

The curves in the bottom part of each panel in Figure 10 show the resulting UV continuum fluxes (left), and the line flux in Ly $\alpha$ (right) according to the three population synthesis models. For reference, the SFRs on which these flux estimates are based are displayed at the top of each panel (compare with the corresponding panel in Figure 2). We also show the total stellar masses at the top of each panel (right-hand axis). Figure 10 shows that the galaxy simulated here would be observable out to redshifts $z \lesssim 13$ in both the UV continuum and the Ly $\alpha$ line in deep surveys with exposures $10^{5}-10^{6} \mathrm{~s}$. While the flux in the UV continuum is insensitive to the properties of the stellar populations, the Ly $\alpha$ flux is significantly larger in the case of metal-free stellar population with top-heavy IMF than in the other two cases. At redshifts higher than $z \gtrsim 13$, the reduction in the SFRs, mostly due to radiative feedback, causes the fluxes to decrease sharply below any practical detection limit.

The results presented here are consistent with our earlier results (Pawlik et al. 2011), after accounting for the differences in the SFRs. We caution that the SFRs on which our estimates are based are uncertain for a number of reasons (see also the discussion in Section 7). First, our simulations do not account for feedback from SNe or chemical enrichment, both of which must alter the SFRs significantly. The SFRs are also uncertain because in the absence of self-regulation by feedback they will depend on the star formation efficiency (e.g., Ricotti et al. 2002), which is currently not well constrained at the high redshifts of interest and for which we have assumed a value appropriate for the local universe (see Section 2.3). As discussed in Section 4.3, the finite resolution of our simulations introduces additional uncertainties in the SFRs. Our work is a step toward a more general discussion of the observability of the first galaxies, which ideally should be based on more sophisticated simulations of a larger galaxy sample (e.g., Ricotti et al. 2008).

\section{DISCUSSION}

The two nested gas disks in our simulations form only after the halo has reached a virial temperature significantly larger than $10^{4} \mathrm{~K}$. In light of the scale-free nature of cold dark matter structure formation, this relatively late formation of the disks may be surprising. However, it is consistent with results from previous zoomed simulations of the first atomically cooling galaxies which have not exhibited orderly rotation of the gas (e.g., Wise et al. 2008; Greif et al. 2008; Prieto et al. 2013). On the other hand, the occurrence of the disks in the dwarf-sized halos fits smoothly in line with simulations of galaxies more massive than the first atomically cooling galaxies assembling at lower redshifts (e.g., Mashchenko et al. 2008; Pawlik et al. 2011; Romano-Díaz et al. 2011; Wise et al. 2012).

The results from these previous works may point at a threshold halo mass for the formation of the first disk galaxies in the range $\sim 10^{8}-10^{10} M_{\odot}$ at $z \sim 10$. This mass scale is similar to the mass scale above which stellar feedback becomes inefficient. However, the fact that simulations of the first atomically cooling galaxies have yielded a turbulent morphology even in the absence of star formation suggests that the two scales are physically unrelated. Indeed, in our simulations, the halo mass at the time of disk formation is insensitive to the inclusion of feedback. Nevertheless, the increased robustness of dwarf galaxies against stellar feedback helps to preserve the disks (e.g., Kaufmann et al. 2007). We caution that properties other than halo mass such as, e.g., the environment, or the merger 
history, may be critical to disk formation in the first galaxies (Prieto et al. 2013).

Our results imply that galaxies with SFRs of $\sim 0.1 M_{\odot} \mathrm{yr}^{-1}$ will be among the faintest galaxies JWST is likely to detect at $z>10$, confirming earlier estimates (e.g., Haiman \& Loeb 1998; Oh 1999; Tumlinson et al. 2001; Ricotti et al. 2008; Zackrisson et al. 2011; Pawlik et al. 2011). According to our simulations, such galaxies reside in halos with masses of $\sim 10^{9} M_{\odot}$ having stellar masses of $\sim 10^{7} M_{\odot}$. In principle, JWST is sufficiently powerful to detect the light from stellar clusters with masses as low as $10^{5}-10^{6} M_{\odot}$ (e.g., Johnson et al. 2009; Zackrisson et al. 2011; Pawlik et al. 2011). Our simulations do not support the formation of such massive clusters, which would require local SFRs $\sim 1 M_{\odot} \mathrm{yr}^{-1}$, an order of magnitude higher than found here. However, star formation in our simulations is unresolved, and it is possible that star formation in the first galaxies is more clustered or bursty than implied here. In this case, JWST could detect galaxies inside halos less massive than considered here, or inside halos that are more strongly affected by feedback than suggested by our simulations. On the other hand, because we do not resolve the formation of stars from first principles, galaxies could be less efficient star-formers than implied by our simulations, and hence be fainter. Such fainter galaxies may still be seen if they are gravitationally lensed (e.g., Zackrisson et al. 2012).

Our simulations have ignored a potentially very important physical process, namely the explosion of massive stars in SNe. SNe can provide a strong negative feedback by heating and expelling gas from even relatively massive halos (e.g., Mac Low \& Ferrara 1999). Previous works have shown that SN feedback can suppress star formation strongly and lead to bursty star formation histories (e.g., Stinson et al. 2007). SN feedback further may create a highly spatially inhomogeneous medium, likely enhancing the fraction of low column density sight lines and hence the fraction of escaping ionizing photons (e.g., Yajima et al. 2009; but see Dove et al. 2000). SN feedback may disturb the assembly of disks inside the first galaxies strongly (e.g., Wise et al. 2012; but see, e.g., Mashchenko et al. 2008). Moreover, SN feedback may modify the structure of the dark matter halos significantly (e.g., Mashchenko et al. 2008; Governato et al. 2012; Brooks \& Zolotov 2012; Garrison-Kimmel et al. 2013).

Our simulations also ignored the chemical enrichment of the gas by the metals synthesized in stars. Simulations that track the production and transport of metals suggest that the transition between metal-free and metal-enriched stellar populations may occur early in the history of the universe (e.g., Tornatore et al. 2007; Maio et al. 2010; Wise et al. 2012). Significant uncertainties, however, remain as to the efficiency of the mixing of metals with the primordial gas and the level of spatial homogeneity of metal enrichment (e.g., Scannapieco et al. 2002; Ricotti et al. 2008). This, together with the fact that not all stars are expected to explode in SNe and enrich the gas but may instead collapse directly into black holes (e.g., Heger et al. 2003), leaves open the possibility of the formation of metal-free stars in select regions of the universe down to relatively low redshifts (e.g., Tornatore et al. 2007; Trenti et al. 2009; Johnson 2010; Fumagalli et al. 2011; Simcoe et al. 2012). However, even if all stars would collapse into black holes without $\mathrm{SNe}$, feedback from accretion onto the black holes may still affect the evolution of the galaxies in a manner not captured by our simulations (e.g., Ricotti \& Ostriker 2004; Alvarez et al. 2009; Jeon et al. 2012).

\section{SUMMARY}

We have presented cosmological SPH simulations of a dwarf galaxy assembling in a halo reaching $10^{9} M_{\odot}$ at $z=10$. The simulations were identical to our earlier simulations of such a galaxy in that they followed the non-equilibrium chemistry and cooling of primordial gas. They improved on our earlier simulations by including the formation of massive metal-free stars. To investigate the radiative feedback from these stars, we compared a simulation in which star particles emitted both molecular hydrogen dissociating and hydrogen/helium ionizing radiation and a simulation in which star particles emitted only dissociating radiation with a simulation inside which star particles remained dark.

Our main results are as follows.

1. Dissociating and ionizing radiation exert a strong negative feedback by suppressing star formation in the main minihalo progenitor of the dwarf galaxy, but have little effect on star formation as soon as the progenitor evolves into an atomically cooling galaxy.

2. Radiative feedback suppresses the central dark matter densities in the dwarf galaxy main progenitor minihalo relative to the densities found in the simulation without radiation. The dark matter density profile of the dwarf galaxy is singular isothermal independent of the inclusion of radiation shortly after the minihalo has evolved into an atomic cooling halo.

3. The dwarf galaxy halo hosts two nested disks below $z \lesssim 12.5$. The formation history and structure of the disks are insensitive to the inclusion of dissociating and ionizing radiation. These results are consistent with a picture in which the first disk galaxies form inside dark matter halos with masses $\gtrsim 10^{9} M_{\odot}$ at $z \gtrsim 10$.

4. The inclusion of dissociating and ionizing radiation lowers the baryon fractions inside the minihalos in the neighborhood of the dwarf galaxy. The baryon fractions are lowest in minihalos with masses $\lesssim 10^{6} M_{\odot}$, a consequence of Jeans filtering and photoevaporation from external ionizing sources, and in minihalos with masses $\sim 10^{7}-10^{8} \mathrm{M}_{\odot}$, here primarily a consequence of photoevaporation of gas by internal ionizing sources.

5. Galaxies with SFRs $\sim 0.1 M_{\odot} \mathrm{yr}^{-1}$ will be among the faintest galaxies the upcoming JWST will detect in deep exposures of the $z \gtrsim 10$ universe. Our simulations suggest that such galaxies reside in halos with masses $\sim 10^{9} M_{\odot}$ and have stellar masses $\sim 10^{7} M_{\odot}$.

We caution that our conclusions are subject to statistical uncertainties implied by the small volume of the high-resolution region in our simulations. Another major shortcoming of our simulations is the lack of feedback from SN explosions. Such feedback can potentially have a significant impact on the evolution of low-mass galaxies. Feedback from $\mathrm{SNe}$ may heavily disturb the assembly of disks, and strongly decrease the SFRs inside dwarf galaxies, thus affecting also estimates of their observability. Our simulations also did not account for the chemical enrichment of the interstellar and intergalactic gas and the associated transition from metal-free to metal-enriched stars. The effects of SN feedback and chemical enrichment are left to be investigated in future work.

We are grateful to Volker Springel, Joop Schaye, and Claudio Dalla Vecchia for letting us use their versions of GADGET as well as their implementations of FOF and SUBFIND. We thank 
the referee for the constructive comments which improved the discussion of the present work. A.H.P. thanks Joop Schaye, Claudio Dalla Vecchia, Alireza Rahmati, Milan Raičević, Jacob Hummel, and Marcel Haas for helpful discussions. The simulations presented here were carried out at the Texas Advanced Computing Center (TACC). This work also benefited from the use of supercomputer facilities of the National Computing Facilities Foundation (NCF). This research is supported by NASA through Astrophysics Theory and Fundamental Physics Program grant NNX09AJ33G and through NSF grant AST1009928. A.H.P. receives funding from the European Union's Seventh Framework Programme (FP7/2007-2013) under grant agreement number 301096-proFeSsOR.

\section{APPENDIX}

In this appendix, we discuss our implementation of photoionization and photoheating by stellar sources (Appendix A.1). We describe the implementation of the RT (Appendix A.1.1), the treatment of multi-frequency radiation (Appendix A.1.2), the computation of the photoionization and photoheating rates (Appendix A.1.3), and the coupling of the RT to the hydrodynamical evolution (Appendix A.1.4). We also discuss tests of our RT implementation (Appendix A.2), specifically addressing the coupling of the RT with the solver for the chemical and thermal evolution (Appendix A.2.1), and with the hydrodynamics (Appendix A.2.2). We emphasize that the approximations and choices of numerical parameters employed in this work and described below are specific to the current work.

\section{A.1. Implementation}

\section{A.1.1. TRAPHIC}

The RT makes use of the RT code TRAPHIC (Pawlik \& Schaye 2008, 2011) implemented in a customized copy of version 3 of GADGET (Springel 2005; Schaye et al. 2010). In simulations with TRAPHIC, the RT equation is solved by tracing a finite number of discrete photon packets emitted by ionizing source particles through the simulation box. This is done directly on the irregular grid defined by the SPH particles, at the speed of light, and in a photon-conserving manner (Abel et al. 1999). In addition to the description of TRAPHIC given below, the reader may refer to the original publications for further details.

Each photon packet carries photons of characteristic frequency $v$. We denote the total number of frequency bins used to discretize the radiation spectrum by $N_{v}$. The transport of photons during a single time step proceeds by a succession of emission and transmission steps that move photon packets from individual particles, either SPH or star particles, to a number of $\tilde{N}_{\text {ngb }}$ SPH neighbors. The neighbors of a given particle are particles located inside the neighbor sphere, which is a sphere centered on the particle and contains $\tilde{N}_{\text {ngb }}$ neighboring particles. Therefore, $\tilde{N}_{\text {ngb }}$ determines the spatial resolution at which the RT is carried out. We choose $\tilde{N}_{\text {ngb }}=48$, which reflects a compromise between keeping high spatial resolution and controlling particle discreteness noise (Pawlik \& Schaye 2008).

Star particles emit photon packets to their $\tilde{N}_{\text {ngb }}$ neighboring SPH particles inside $N_{\mathrm{EC}}$ emission cones, each subtending a solid angle of $4 \pi / N_{\mathrm{EC}}$. Emission cones tessellate the sky and are used to accomplish the isotropic emission of photon packets to the SPH neighbors despite the possibly highly anisotropic distribution of the SPH particles (see the Appendix in Pawlik $\&$ Schaye 2008). Photon packets emitted inside emission cones containing multiple SPH neighbors are split in proportion to the inverse squared distance between the source and the neighbors to account for the dilution of the radiation field with distance from the source. The central axes of the emission cones define the initial propagation directions of the emitted photon packets. The parameter $N_{\mathrm{EC}}$ determines the angular sampling of the sky as seen from any given ionizing source. We choose $N_{\mathrm{EC}}=8$. Even though the orientation of the emission cone tessellation is randomly rotated at each RT time step, our choice of a small number of emission cones may imply increased random scatter in the distribution of the photoionized and photoheated gas, especially around halos that contain only a few star particles (see Appendix A.2.3 for an illustration).

SPH particles that receive photon packets transmit these packets along the associated propagation directions to their downstream neighbors. Individual photon packets are transmitted only to the SPH neighbors located inside transmission cones, which are regular cones with solid angles subtending $4 \pi / N_{\mathrm{TC}}$ steradians centered around the propagation directions and with their apex attached to the transmitting particle, where $N_{\mathrm{TC}}$ is a parameter specified below. The use of transmission cones prevents uncontrolled diffusion of photon packets on the set of irregularly distributed SPH particles and keeps the photon transport directed. Photon packets that are emitted inside transmission cones containing multiple SPH neighbors are split equally between these neighbors.

The parameter $N_{\mathrm{TC}}$ sets the angular resolution at which the RT is performed. Because the transmission cones are defined locally at the positions of the transmitting SPH particles and because photon packets are only distributed among the subset of the $\tilde{N}_{\text {ngb }}$ neighbors that fall inside these transmission cones, the angular resolution is independent of the distance from the sources. In this work we adopt $N_{\mathrm{TC}}=128$. In test simulations of the RT around multiple sources inside a static cosmological density field presented in Pawlik \& Schaye (2008) we found that the results had converged at a lower angular resolution of $N_{\mathrm{TC}}=32$. Note that virtual particles are created to accomplish the emission and transport of photon packets in cones that do not contain SPH neighbors, as described in Pawlik \& Schaye (2011).

Photon packets received by SPH particles are merged, which allows one to control the number of photon packets inside the simulation box and to avoid the scaling of the computational cost with the number of sources. The merging is done by binning photon packets in solid angle using a set of $N_{\mathrm{RC}}$ tessellating reception cones with solid angles $4 \pi / N_{\mathrm{RC}}$ attached to each SPH particle. The merging is done separately for each frequency bin and hence it limits the number of photon packets to be transmitted next to at most $N_{\mathrm{RC}} \times N_{v}$. Accordingly, the computational cost of the RT scales with $N_{\mathrm{RC}}$, but not with the number of ionizing sources. In this work we set $N_{\mathrm{RC}}=8$, motivated by test simulations similar to those presented in Pawlik \& Schaye (2011).

Photon packets are associated with a clock that is used to control the speed at which they travel (see Pawlik \& Schaye 2008). In this work we set this speed equal to the physical speed of light. However, for computational efficiency we allow photon packets to travel only a single inter-particle distance during individual RT time steps. In the limit of small RT time steps, transporting photon packets at the speed of light but limited by a single inter-particle distance is equivalent to solving the time-dependent RT equation (see Pawlik \& Schaye 2008 for a similar argument in the case of the time-independent RT). 
The hybrid approach employed here prevents the photons from traveling faster than the speed of light but, depending on the size of the RT time step and the inter-particle distance, may imply that photons travel at an effective speed that is lower than the speed of light, possibly resulting in an artificially delayed propagation of ionization fronts. We will discuss the effects of this approximation in Appendix A.2.3.

\section{A.1.2. Absorption of Ionizing Photons by Hydrogen/Helium}

A fraction $1-\exp [-\tau(\nu)]$ of each photon packet in frequency bin $v$ emitted or transmitted from particle $i$ at position $\mathbf{r}_{i}$ to SPH neighbor $j$ at position $\mathbf{r}_{j}$, separated by the propagation distance $d_{i j}$, is absorbed. The optical depth $\tau(v)$ is the sum $\tau(v)=\sum \tau_{\alpha}(v)$ of the optical depths $\tau_{\alpha}(v)$ of each absorbing species $\alpha \in\left\{\mathrm{HI}_{\mathrm{I}}\right.$, He I, He II $\}$, and $\tau_{\alpha}(\nu)=\int_{\mathbf{r}_{i}}^{\mathbf{r}_{j}} d r \sigma_{\alpha}(\nu) n_{\alpha}(\mathbf{r}) \approx$ $\sigma_{\alpha}(\nu) n_{\alpha}\left(\mathbf{r}_{j}\right) d_{i j}$. The number of photons absorbed by species $\alpha$ is $\delta N_{\mathrm{abs}, \alpha}(\nu)=\left[w_{\alpha}(\nu) / \sum_{\alpha} w_{\alpha}(\nu)\right] \delta N_{\text {abs }}(\nu)$, where the weights $w_{\alpha}(\nu)=\tau_{\alpha}(\nu)$ (Pawlik \& Schaye 2011) and $\delta N_{\text {abs }}(\nu)=$ $\sum_{\alpha} \delta N_{\text {abs, } \alpha}(v)$ is the total number of absorbed photons.

In this work we choose, for reasons of computational efficiency, to transport hydrogen-ionizing radiation using a single frequency bin, i.e., we set $N_{v}=1$. In this bin, we adopt frequency-averaged photoionization cross-sections $\left\langle\sigma_{\gamma \alpha}\right\rangle$ for the absorption by the individual species $\alpha$ using the gray approximation (e.g., Mihalas \& Weibel Mihalas 1984),

$$
\left\langle\sigma_{\gamma \alpha}\right\rangle \equiv \int_{\nu_{\alpha}}^{\infty} d \nu \frac{4 \pi J_{v}(v)}{h_{\mathrm{P}} v} \sigma_{\gamma \alpha}(\nu) \times\left[\int_{\nu_{\mathrm{H} \mathrm{I}}}^{\infty} d \nu \frac{4 \pi J_{v}(v)}{h_{\mathrm{P}} v}\right]^{-1}
$$

where $J_{v}(v)$ is the mean intensity of the radiation field and $h_{\mathrm{P}} v_{\alpha}$ is the ionization potential. We assume that the mean intensity $J_{v}(v)$ is characterized by a blackbody spectrum of temperature $T_{\mathrm{BB}}=10^{5} \mathrm{~K}$, appropriate for the emission of radiation by the first stars (e.g., Schaerer 2003), which gives $\left\langle\sigma_{\gamma \mathrm{H}_{\mathrm{I}}}\right\rangle=1.63 \times 10^{-18} \mathrm{~cm}^{2},\left\langle\sigma_{\gamma \mathrm{He}_{\mathrm{I}}}\right\rangle=2.28 \times 10^{-18} \mathrm{~cm}^{2}$, and $\left\langle\sigma_{\gamma \text { He II }_{\text {II }}}\right\rangle=6.19 \times 10^{-20} \mathrm{~cm}^{2}$. We have employed the fits to the photoionization cross-sections from Verner et al. (1996).

At fixed mean intensity $J_{v}(v)$, the gray approximation implies photoionization rates identical to those inferred from a full multi-frequency treatment. However, because of the use of only a single frequency bin, our simulations ignore the hardening of the radiation spectrum with distance from the source caused by the preferential absorption of lower energy photons with larger absorption cross sections (see, e.g., the discussion in Pawlik \& Schaye 2011).

\section{A.1.3. Photoionization and Photoheating Rates}

At the end of each RT time step the photoionization and photoheating rates are computed. We determine the photoionization rates directly from the total number of photons $N_{\mathrm{abs}, \alpha}(\nu)=$ $\sum \delta N_{\mathrm{abs}, \alpha}(\nu)$ absorbed by a given SPH particle during the RT time step $\Delta t_{\mathrm{r}}$ in frequency bin $v$,

$$
\Gamma_{\gamma \alpha}=\frac{\sum_{\nu} N_{\mathrm{abs}, \alpha}(v)}{\eta_{\alpha} N_{\mathrm{H}} \Delta t_{\mathrm{r}}}
$$

where $N_{\mathrm{H}} \equiv m_{\mathrm{gas}} X_{\mathrm{H}} / m_{\mathrm{H}}$ is the number of hydrogen atoms associated with the SPH particle of mass $m_{\text {gas }}$. This ensures that the same number of photons that have been removed from the simulation is used to determine the ionization balance and temperature of the ionized gas, i.e., photon conservation (Abel et al. 1999).
The heating rate per atom due to photoionization of species $\alpha$, assuming a single frequency bin in the gray approximation, is $\mathcal{E}_{\gamma \alpha}=\Gamma_{\gamma \alpha}\left\langle\epsilon_{\alpha}\right\rangle$, where

$$
\begin{aligned}
\left\langle\epsilon_{\alpha}\right\rangle= & \int_{v_{\alpha}}^{\infty} d v \frac{4 \pi J_{v}(v)}{h_{\mathrm{P}} v} \sigma_{\gamma \alpha}(v)\left(h_{\mathrm{P}} v-h_{\mathrm{P}} v_{\alpha}\right) \\
& \times\left[\int_{v_{\alpha}}^{\infty} d v \frac{4 \pi J_{\nu}(v)}{h_{\mathrm{P}} v} \sigma_{\gamma \alpha}(v)\right]^{-1}
\end{aligned}
$$

is the average energy of the absorbed ionizing photons in excess of the photoionization threshold. Because we assume that the mean intensity $J_{v}(v)$ is characterized by a blackbody spectrum of temperature $T_{\mathrm{BB}}=10^{5} \mathrm{~K}$, the average excess energies for photoionization of hydrogen and helium are $\left\langle\epsilon_{\mathrm{HI}}\right\rangle=6.32 \mathrm{eV}$, $\left\langle\epsilon_{\mathrm{He}}\right\rangle=8.70 \mathrm{eV}$, and $\left\langle\epsilon_{\mathrm{He} \text { II }}\right\rangle=7.88 \mathrm{eV}$.

\section{A.1.4. Radiation-Hydrodynamical Coupling}

The radiation-hydrodynamical evolution of the gas is followed by invoking a series of subcycles to compute the dynamics, RT, chemistry, cooling, and heating of the gas. The dynamical evolution of the gas is followed on the gravitohydrodynamical time steps set by the standard time integration scheme of the GADGET code (Section 2.1). The RT is performed by subcycling the smallest (among all particles) gravito-hydrodynamical time step $\Delta t_{\mathrm{s}}$, with RT time steps of size $\Delta t_{\mathrm{r}}$. Unless stated otherwise, we adopt $\Delta t_{\mathrm{r}}=\min \left[\Delta t_{\mathrm{s}}, 0.1 \mathrm{Myr}\right]$. We assume that the chemical abundances and temperatures do not change during a single RT time step.

Chemistry, heating, and cooling of the gas are computed at the end of each RT time step. This is done by subcycling the RT time step using time steps computed by the implicit non-equilibrium solver described in Section 2.2. Note that because photoionization rates are obtained from the number of absorptions computed under the assumption that species fractions and temperatures remain constant during the time $\Delta t_{\mathrm{r}}$, not all photons that have been absorbed always end up consumed in the computation of the chemistry and thermodynamics of the gas. This is a consequence of the evolution of the chemical composition and temperature during the subcycling. To ensure photon conservation we reinsert the remaining photons into the RT at the beginning of the next RT time step, and set them to be propagated along their original directions.

The change in chemical abundances and temperature affects the dynamics of the particles only at the end of their gravitohydrodynamical time steps, at which time the particle entropies are updated and the sizes of the new gravito-hydrodynamical time steps are determined. In GADGET, particles evolve along a hierarchy of individual particle gravito-hydrodynamical time steps that may be much longer than the smallest such time step, the latter also being the time step at which the radiative cooling and heating are computed. This means that the radiation-hydrodynamical response of the gas to photoionization heating may occur with a delay. Recently, Durier \& Dalla Vecchia (2012) have shown, in the context of thermal feedback from $\mathrm{SN}$ explosions, that the lack of a prompt response to localized energy injection may lead to a strong violation of the conservation of energy when using the standard GADGET time integration scheme employed also here. Our simulations may suffer from similar numerical artifacts.

\section{A.2. Tests}

We have previously described a number of tests of our implementation of TRAPHIC in GADGET for the RT on static density 
fields. In Pawlik \& Schaye (2008), we carried out monochromatic RT simulations of increasing complexity, assuming that the gas is composed only of hydrogen and has a fixed temperature. By comparing with reference solutions such as those published in Iliev et al. (2006), we demonstrated the ability of TRAPHIC to accurately capture the evolution of ionization fronts, and to reproduce the ionized fractions. We also showed that TRAPHIC is able to produce sharp shadows behind opaque absorbers, which gives rise to the typical "butterfly" shape of the ionized regions (e.g., Abel et al. 1999). In Pawlik \& Schaye (2011), we extended our implementation of TRAPHIC to enable the transport of multi-frequency radiation, to account also for the ionization of helium, and to compute the evolution of the gas temperature due to radiative cooling and photoionization heating. Results from test simulations with this new implementation were in excellent agreement with reference solutions, such as those in Iliev et al. (2006).

The implementation of TRAPHIC in GADGET that we use in this work differs from that described in Pawlik \& Schaye (2008, 2011) in two main respects. First, we have substituted the explicit solver for the evolution of the chemistry and temperature of primordial atomic gas in the presence of photoionizations used and tested in Pawlik \& Schaye (2011) with the implicit solver used in Pawlik et al. (2011) and described in Section 2.2. This solver accounts for additional species, such as molecular hydrogen, and employs different rates for the atomic and molecular physics. It has been described and extensively tested in a number of publications (e.g., Johnson \& Bromm 2006; Greif et al. 2010). However, it has not yet been tested in combination with TRAPHIC. In Appendix A.2.1, we therefore repeat the RT test simulation on a static cosmological density field from Pawlik \& Schaye (2011). The results of this test are in excellent agreement with our previous results, demonstrating the validity of our implementation.

Second, the current work is the first to employ TRAPHIC in radiation-hydrodynamic simulations that account for the feedback of photoionization heating on the gas dynamics. We have performed the radiation-hydrodynamical tests from Iliev et al. (2009), and we have achieved excellent agreement with the published reference results in all of them. In Appendix A.2.2 we provide a discussion of one of the tests relevant to the present work, the simulation of the evaporation of a minihalo by an internal ionizing source.

Finally, in Appendix A.2.3, we investigate how our adoption of a limited photon propagation speed and a finite angular sampling affects the early evolution of the minihalo in simulation $L W+R T$ analyzed in the main text.

\section{A.2.1. Radiative Transfer and Chemical and Thermal Evolution}

In this appendix, we repeat Test 4 of the cosmological RT code comparison project (Iliev et al. 2006) that we have previously discussed in Pawlik \& Schaye (2011), using a chemistry solver different from the one employed here. The test involves the simulation of $\mathrm{H}$ II regions around multiple ionizing sources in a static cosmological density field. It was designed to capture important aspects of state-of-the-art simulations of hydrogen reionization, such as the delayed propagation in or trapping of ionization fronts by dense gas.

The setup of this test is identical to that of Test 4 in Pawlik $\&$ Schaye (2011), to which we refer the reader for a detailed description. Briefly, the initial conditions are provided by a snapshot (at redshift $z \approx 8.85$ ) from a cosmological $N$-body and gas-dynamical uniform-mesh simulation. The simulation box is
$L_{\text {box }}=0.5 h^{-1} \mathrm{Mpc}$ comoving on a side, where $h=0.7$, and is uniformly divided into $N_{\text {cell }}=128^{3}$ cells. We Monte Carlo sample this density field to replace the mesh cells with $N_{\mathrm{SPH}}=$ $N_{\text {cell }}=128^{3} \mathrm{SPH}$ particles. The gas consists purely of atomic hydrogen and is assumed to be initially neutral at temperature $T=100 \mathrm{~K}$. The ionizing sources are chosen to correspond to the 16 most massive halos in the box, and are assumed to have blackbody spectra with temperature $T_{\mathrm{BB}}=10^{5} \mathrm{~K}$.

For comparison with Pawlik \& Schaye (2011) we solve the time-independent RT equation with an angular resolution of $N_{\mathrm{c}}=32$, set the number of neighbors to which sources emit radiation to $\tilde{N}_{\text {ngb }}=32$, employ a time step $\Delta t_{\mathrm{r}}=10^{-4} \mathrm{Myr}$, and transport photons only over a single inter-particle distance per time step. We transport radiation using a single frequency bin, employing the gray photoionization cross-section $\left\langle\sigma_{\gamma \mathrm{H}_{\mathrm{I}}}\right\rangle=$ $1.63 \times 10^{-18} \mathrm{~cm} \mathrm{~s}^{-1}$. We assume that each photoionization adds $\left\langle\epsilon_{\mathrm{H}}\right\rangle=6.32 \mathrm{eV}$ to the thermal energy of the gas, as appropriate for the adopted blackbody spectrum.

In Figure 11, we show results of this simulation and compare them with those presented in Pawlik \& Schaye (2011) and also with the reference results reported in Iliev et al. (2006). The agreement is very good if one accounts for the differences in the atomic physics and the approximations employed (see Pawlik \& Schaye 2011 for a detailed discussion).

\section{A.2.2. Radiation-Hydrodynamical Coupling}

In this appendix, we perform Test 6 of the cosmological RT code comparison project (Iliev et al. 2009). This test consists of the simulation of the expansion of an $\mathrm{H}$ II region driven by an ionizing point source at the center of a spherically symmetric region with a steeply declining gas density profile. In contrast to the test described in the previous appendix, the gas distribution is not forced to remain static but may evolve in response to photoionization heating from the central source. The test situation shares some of the main features of the evolution of gas densities inside high-redshift cosmological minihalos under photoionization from a central massive metal-free star.

The physical setup of the test is as follows. The initial gas densities are described by a cored isothermal density profile,

$$
n_{\mathrm{H}}(r)= \begin{cases}n_{0} & \text { if } r \leqslant r_{0} \\ n_{0}\left(r / r_{0}\right)^{-2} & \text { otherwise }\end{cases}
$$

We follow Iliev et al. (2009) and set $n_{0}=3.2 \mathrm{~cm}^{-3}$ and $r_{0}=$ $91.5 \mathrm{pc}$, and we assume that the gas, consisting solely of atomic hydrogen, is initially neutral at temperature $100 \mathrm{~K}$. The central source has a constant ionizing luminosity of $10^{50}$ photons s ${ }^{-1}$ throughout the simulation, and is characterized by a blackbody spectrum with temperature $10^{5} \mathrm{~K}$. Following Iliev et al. (2009), all relevant cooling processes are included, except for Compton cooling. In this test, gravitational forces are ignored.

We implement the power-law density profile by applying a local radial stretch along the direction toward the central source to the inter-particle distances of SPH particles that are initially distributed uniformly with density $\tilde{n}_{\mathrm{H}}$. Denoting the initial particle positions with $(\tilde{r}, \theta, \phi)$, such a stretching is expressed by the transformation $(\tilde{r}, \theta, \phi) \rightarrow(r, \theta, \phi)$, where $\theta$ and $\phi$ are the usual two angles needed to specify a position in spherical coordinates. Mass conservation then requires the new coordinates $(r, \theta, \phi)$ to satisfy

$$
n_{\mathrm{H}}(r) r^{2} \sin \theta d r d \theta d \phi=\tilde{n}_{\mathrm{H}} \tilde{r}^{2} \sin \theta d \tilde{r} d \theta d \phi
$$



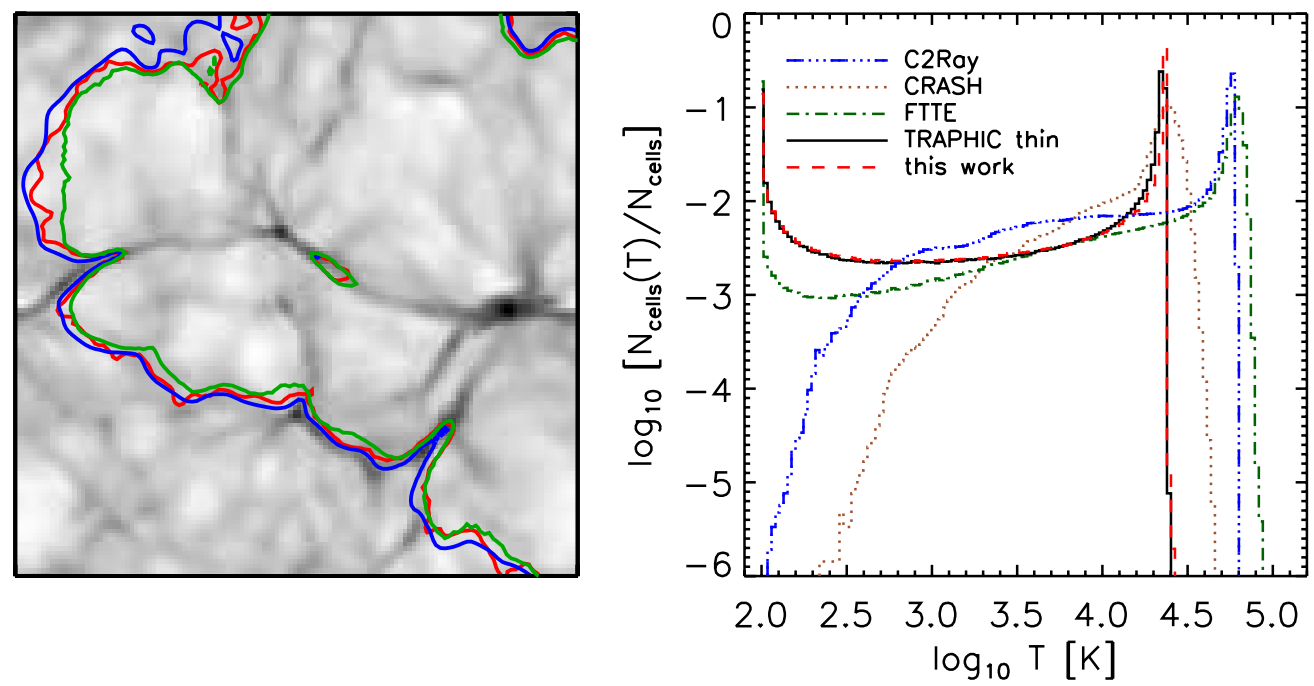

Figure 11. Test 4: cosmological reionization by multiple stellar sources (Iliev et al. 2006). Left: thin slice through the center of the simulation box at time $t=0.2 \mathrm{Myr}$, showing contours of neutral hydrogen fraction $\eta_{\mathrm{HI}}=0.5$, on top of the density field (gray image). The ionization fronts obtained in this work (red) are in very good agreement with the reference results obtained using C2RAY (blue; Mellema et al. 2006) and CRASH (green; Ciardi et al. 2001; Maselli et al. 2003, 2009). Right: histograms of the gas temperature at $t=0.2 \mathrm{Myr}$. The results obtained in this work are in excellent agreement with those presented in Pawlik \& Schaye (2011, histogram labeled TRAPHIC thin for reference with our earlier work) obtained with a different solver for the chemical and thermal evolution. They are in very good agreement with the reference solutions obtained with C2RAY, CRASH, and FTTE (Razoumov \& Cardall 2005) as reported in Iliev et al. (2006), if one accounts for the differences in the atomic physics and the approximations employed.

(A color version of this figure is available in the online journal.)

Substituting a power-law density profile, $n_{\mathrm{H}}(r) \propto r^{n}$, the last equation can be integrated to yield $r \propto \tilde{r}^{3 /(3-n)}$. In the present case, $n=2$, and hence $r \propto \tilde{r}^{3}$. Therefore, a singular isothermal profile can be obtained by stretching the initial inter-particle distances between uniformly distributed particles along the radial directions toward the center by a factor $\tilde{r}^{2}$. The initial uniform density field is generated by placing particles randomly in the simulation box. To reduce the associated shot noise, the resulting particle distribution is regularized by evolving the particles under the influence of a reversed-sign (i.e., repulsive) gravitational force until the particles settle down into a glass-like quasi-equilibrium (White 1996). After generating the singular isothermal density profile, we replace the central region within $r_{0}$ with a uniform glass-like particle distribution to introduce the central core.

The numerical parameters used in this test are as follows. We place the ionizing source in the center of a box with linear size $1.6 \mathrm{kpc}$. We sample the gas inside the core with radius $r_{0}$ using 30,000 particles, which corresponds to an equivalent uniform grid resolution of $160^{3}$ cells. This resolution is slightly higher than the uniform grid resolution employed in Iliev et al. (2006), which was $128^{3}$ cells. We use an angular resolution $N_{\mathrm{c}}=8$, a number of neighbors for the emitting source $\tilde{N}_{\text {ngb }}=32$, employ an RT time step $\Delta t_{\mathrm{r}}=10^{-5} \mathrm{Myr}$, and transport photons only over a single inter-particle distance per time step. We limit the sizes of the individual particle hydrodynamical time steps to be less than $0.1 \mathrm{Myr}$. The radiation is transported using a single frequency bin and assuming a gray photoionization crosssection $\left\langle\sigma_{\gamma \mathrm{H}_{\mathrm{I}}}\right\rangle=1.63 \times 10^{-18} \mathrm{~cm} \mathrm{~s}^{-1}$, as well as that each photoionization adds $\left\langle\epsilon_{\mathrm{HI}_{\mathrm{I}}}\right\rangle=6.32 \mathrm{eV}$ to the thermal energy of the gas. The parameter values are chosen to make contact with the conditions in the simulations presented in the main part of this paper, and to ensure numerical convergence. The test results are not critically sensitive to the adopted parameter values. However, we note that because of our choice of emitting photon packets only once per RT time step, the angular sampling depends on the RT time step, and a larger RT time step would imply an increased scatter in the ionized fraction, temperature, and density profiles (see Appendix A.2.3).

The results of the test are shown and discussed in Figure 12. The results obtained with TRAPHIC are in very good agreement with the reference results published in Iliev et al. (2009).

\section{A.2.3. Effect of Limited Photon Packet Propagation Speed and Finite Angular Sampling}

In this appendix, we discuss the effects of our approximations regarding the limited photon propagation speed and the emission of photon packets by ionizing sources along a finite set of random directions per RT time step. To this end, we have repeated the RT around the first stellar burst in the minihalo progenitor of simulation $L W+R T$. The first test simulation that we have performed, hereafter test-base, employed RT parameters identical to those employed in $L W+R T$. In particular, in this simulation ionizing sources emitted photon packets along $N_{\mathrm{EC}}=8$ random directions per RT time step, and the photon propagation was done at the speed of light but limited to a single inter-particle distance per RT time step. To investigate the effect of the limitation in the propagation speed, simulation test-base is compared with a simulation in which photon packets propagate at the speed of light with no additional limitation regarding the number of inter-particle distances per RT time step (hereafter test-speed; and with ionizing sources emitting photons into $N_{\mathrm{EC}}=8$ random directions per RT time step as in test-base). To investigate the effect of the angular sampling, simulation test-speed is compared with a simulation in which sources emit photon packets along $10^{3}$ times more random directions per RT time step (hereafter test-sampling; and transporting photons at the speed of light as in test-speed). The latter is implemented by repeating the emission of radiation along the $N_{\mathrm{EC}}=8$ random directions $10^{3}$ times per RT time step, with each emission using a different set of $N_{\mathrm{EC}}=8$ random directions and emitting photon packets that contain by a factor $10^{3}$ fewer photons. 

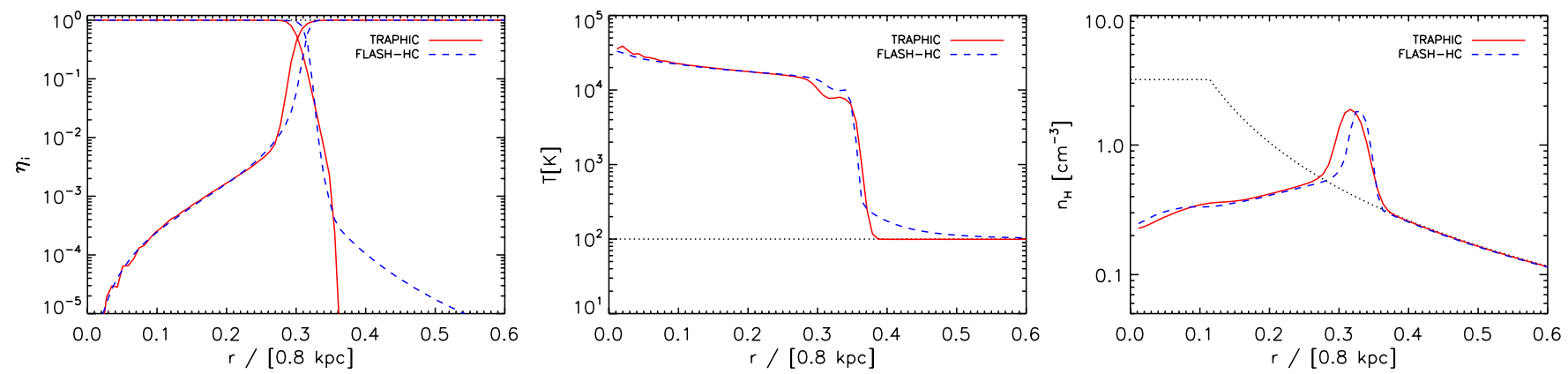

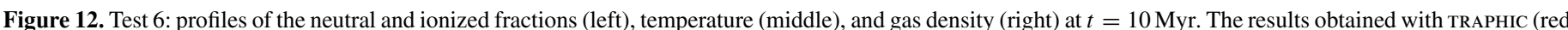

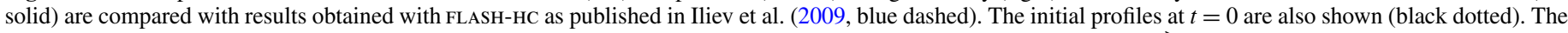

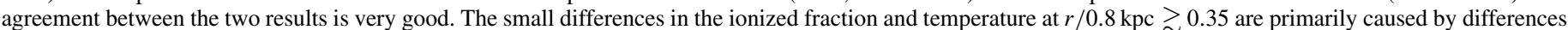

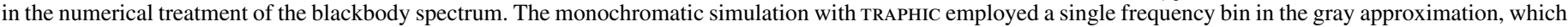

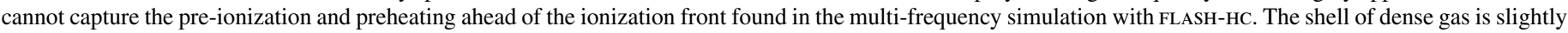

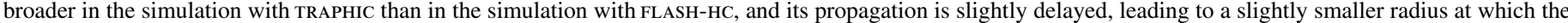

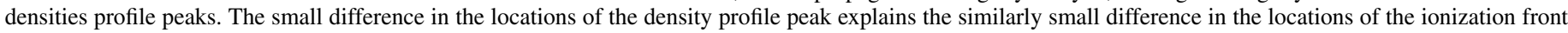

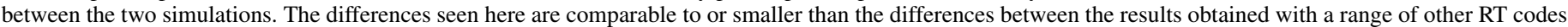
shown in Iliev et al. (2009).

(A color version of this figure is available in the online journal.)
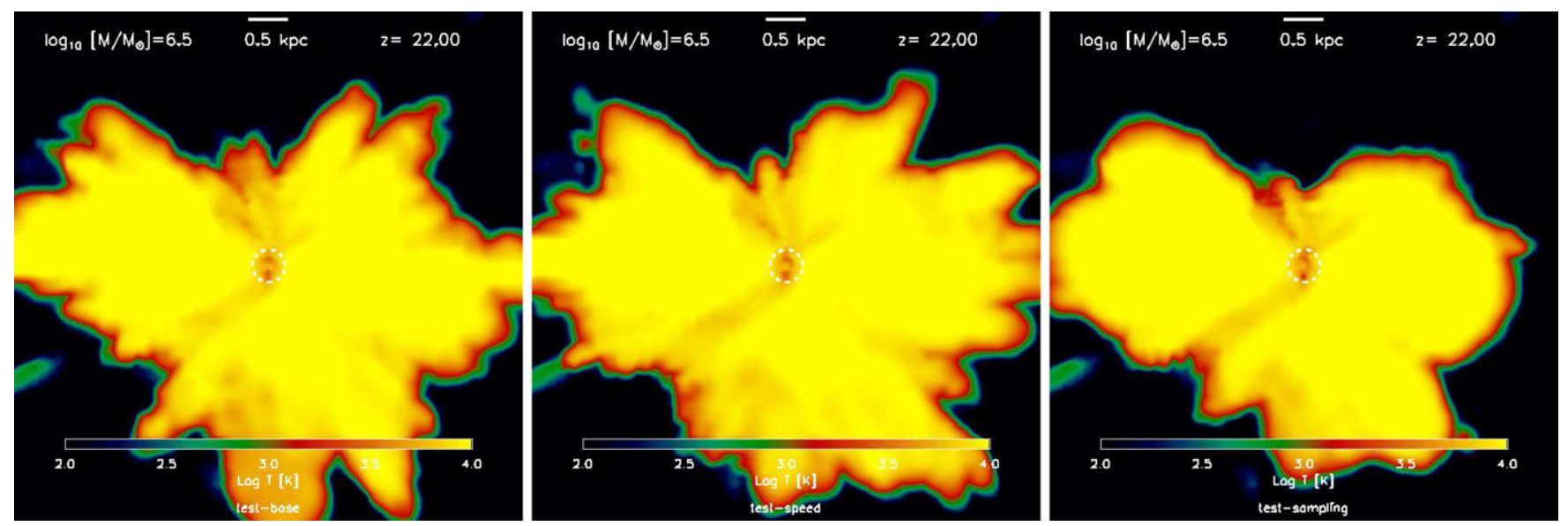

Figure 13. Temperature averaged in a thin slice of thickness $0.2 \mathrm{kpc}$ through the center of the minihalo progenitor at $z=22$ in three simulations that are identical to simulation $L W+R T$ discussed in the main text except for the treatment of the RT. Left: simulation test-base, with a single emission along $N_{\mathrm{EC}}=8$ directions per RT time step and propagation at the speed of light but by at most one inter-particle distance, as in simulation $L W+R T$ discussed in the main text. Middle: simulation test-speed, with a single emission along $N_{\mathrm{EC}}=8$ directions per RT time step and propagation at the speed of light (and no other limitation). Right: simulation test-sampling with $10^{3}$ emissions along $N_{\mathrm{EC}}=8$ random directions per RT step and propagation at the speed of light (and no other limitation). The comparison of simulations test-base (left) and test-speed (middle) shows that the size of the final photoheated region is insensitive to the limit on the distance which photon packets can travel in a given RT time step, thanks to the photon-conserving nature of the RT with TRAPHIC. The comparison of simulations test-speed (middle) and test-sampling (right) shows that an increased angular sampling implemented by emitting photons along an increased number of random directions reduces artifacts in the shape of the photoheated region. Simulation test-sampling shown in the right panel has been used to generate Figure 1.

(A color version of this figure is available in the online journal.)

Figure 13 shows the gas temperature in a thin slice through the center of the minihalo progenitor at $z=22$, at the end of the stellar burst, in the three test simulations test-base (left), test-speed (middle), and test-sampling (right). The final extent of the photoheated region is similar in simulations test-base and test-speed despite the difference in propagation speeds. This is because the RT with TRAPHIC conserves the number of ionizing photons that are transmitted and absorbed, and because the final extent of photoheated $\mathrm{H}$ II regions is set primarily by this number. However, limitations of the speed at which photons are propagated can lead to differences in extent and shape of the developing $\mathrm{H}$ II regions early during their evolution (e.g., Pawlik $\&$ Schaye 2008). The increased angular sampling in simulation test-sampling reduces the noise that characterizes the shape of the photoheated region in simulation test-base, as expected. However, this noise is already small in simulation test-base, which hence yields a photoheated region similar in shape to that in simulation test-sampling. We note (but do not show) that the evolution of the properties of the minihalo, such as, e.g., the maximum gas density, is nearly identical in all three test simulations.

\section{REFERENCES}

Abel, T., Anninos, P., Zhang, Y., \& Norman, M. L. 1997, NewA, 2, 181

Abel, T., Bryan, G. L., \& Norman, M. L. 2002, Sci, 295, 93

Abel, T., Norman, M. L., \& Madau, P. 1999, ApJ, 523, 66

Abel, T., Wise, J. H., \& Bryan, G. L. 2007, ApJL, 659, L87

Ahn, K., Iliev, I. T., Shapiro, P. R., et al. 2012, ApJL, 756, L16

Ahn, K., \& Shapiro, P. R. 2007, MNRAS, 375, 881

Ahn, K., Shapiro, P. R., Iliev, I. T., Mellema, G., \& Pen, U.-L. 2009, ApJ, 695, 1430

Alvarez, M. A., Bromm, V., \& Shapiro, P. R. 2006, ApJ, 639, 621

Alvarez, M. A., Wise, J. H., \& Abel, T. 2009, ApJL, 701, L133

Barkana, R., \& Loeb, A. 1999, ApJ, 523, 54

Barkana, R., \& Loeb, A. 2001, PhR, 349, 125 
Blumenthal, G. R., Faber, S. M., Flores, R., \& Primack, J. R. 1986, ApJ, 301,27

Bouwens, R. J., Illingworth, G. D., Franx, M., et al. 2009, ApJ, 705, 936

Bouwens, R. J., Illingworth, G. D., Oesch, P. A., et al. 2010, ApJL, 708, L69

Bouwens, R. J., Illingworth, G. D., Oesch, P. A., et al. 2012, ApJL, 752, L5

Bovill, M. S., \& Ricotti, M. 2009, ApJ, 693, 1859

Bovill, M. S., \& Ricotti, M. 2011, ApJ, 741, 18

Bovy, J., \& Dvorkin, C. 2012, arXiv:1205.2083

Boylan-Kolchin, M., Bullock, J. S., \& Kaplinghat, M. 2011, MNRAS, 415, L40 Boylan-Kolchin, M., Bullock, J. S., \& Kaplinghat, M. 2012, MNRAS, 422, 1203

Bromm, V., Coppi, P. S., \& Larson, R. B. 2002, ApJ, 564, 23

Bromm, V., Kudritzki, R. P., \& Loeb, A. 2001, ApJ, 552, 464

Bromm, V., \& Larson, R. B. 2004, ARA\&A, 42, 79

Bromm, V., \& Yoshida, N. 2011, ARA\&A, 49, 373

Brooks, A. M., \& Zolotov, A. 2012, arXiv:1207.2468

Brown, P. N., Byrne, G. D., \& Hindmarsh, A. C. 1989, SIAM J. Sci. Stat. Comput., 10, 1038

Bullock, J. S. 2010, arXiv:1009.4505

Bunker, A. J., Stanway, E. R., Ellis, R. S., \& McMahon, R. G. 2004, MNRAS, 355,374

Cai, Z., Fan, X., Jiang, L., et al. 2011, ApJL, 736, L28

Choudhury, T. R., \& Ferrara, A. 2007, MNRAS, 380, L6

Ciardi, B., \& Ferrara, A. 2005, SSRv, 116, 625

Ciardi, B., Ferrara, A., Marri, S., \& Raimondo, G. 2001, MNRAS, 324, 381

Conroy, C., \& Kratter, K. 2012, ApJ, 755, 123

Curtis-Lake, E., McLure, R. J., Dunlop, J. S., et al. 2013, MNRAS, 429, 302

Dijkstra, M., \& Kramer, R. 2012, MNRAS, 424, 1672

Dove, J. B., Shull, J. M., \& Ferrara, A. 2000, ApJ, 531, 846

Dunlop, J. S. 2012, arXiv:1205.1543

Dunlop, J. S., McLure, R. J., Robertson, B. E., et al. 2012, MNRAS, 420, 901

Durier, F., \& Dalla Vecchia, C. 2012, MNRAS, 419, 465

Efstathiou, G. 1992, MNRAS, 256, 43P

Eisenstein, D. J., \& Loeb, A. 1995, ApJ, 443, 11

Finkelstein, S. L., Papovich, C., Giavalisco, M., et al. 2010, ApJ, 719, 1250

Finkelstein, S. L., Papovich, C., Ryan, R. E., Jr., et al. 2012, ApJ, 758, 93

Fumagalli, M., O’Meara, J. M., \& Prochaska, J. X. 2011, Sci, 334, 1245

Furlanetto, S. R., Oh, S. P., \& Briggs, F. H. 2006, PhR, 433, 181

Gardner, J. P., Mather, J. C., Clampin, M., et al. 2006, SSRv, 123, 485

Garrison-Kimmel, S., Rocha, M., Boylan-Kolchin, M., Bullock, J., \& Lally, J. 2013, arXiv: 1301.3137

Glover, S. C. O., \& Brand, P. W. J. L. 2003, MNRAS, 340, 210

Glover, S. C. O., \& Jappsen, A.-K. 2007, ApJ, 666, 1

Gnedin, N. Y., \& Hui, L. 1998, MNRAS, 296, 44

Gnedin, N. Y., Kravtsov, A. V., \& Chen, H.-W. 2008, ApJ, 672, 765

Gnedin, O. Y., Ceverino, D., Gnedin, N. Y., et al. 2011, arXiv:1108.5736

Gnedin, O. Y., Kravtsov, A. V., Klypin, A. A., \& Nagai, D. 2004, ApJ, 616, 16

Governato, F., Zolotov, A., Pontzen, A., et al. 2012, MNRAS, 422, 1231

Greif, T. H., \& Bromm, V. 2006, MNRAS, 373, 128

Greif, T. H., Glover, S. C. O., Bromm, V., \& Klessen, R. S. 2010, ApJ, 716, 510

Greif, T. H., Johnson, J. L., Klessen, R. S., \& Bromm, V. 2008, MNRAS, 387, 1021

Haas, M. R., Schaye, J., Booth, C. M., et al. 2012, arXiv:1211.3120

Haiman, Z., Abel, T., \& Rees, M. J. 2000, ApJ, 534, 11

Haiman, Z., \& Loeb, A. 1998, ApJ, 503, 505

Haiman, Z., Rees, M. J., \& Loeb, A. 1996a, ApJ, 467, 522

Haiman, Z., Rees, M. J., \& Loeb, A. 1997, ApJ, 484, 985

Haiman, Z., Thoul, A. A., \& Loeb, A. 1996b, ApJ, 464, 523

Heger, A., Fryer, C. L., Woosley, S. E., Langer, N., \& Hartmann, D. H. 2003, ApJ, 591,288

Hummel, J. A., Pawlik, A. H., Milosavljevic, M., \& Bromm, V. 2012, ApJ, 755,72

Iliev, I. T., Ciardi, B., Alvarez, M. A., et al. 2006, MNRAS, 371, 1057

Iliev, I. T., Whalen, D., Mellema, G., et al. 2009, MNRAS, 400, 1283

Inoue, A. K. 2011, MNRAS, 415, 2920

Iye, M., Ota, K., Kashikawa, N., et al. 2006, Natur, 443, 186

Jeon, M., Pawlik, A. H., Greif, T. H., et al. 2012, ApJ, 754, 34

Johnson, J. L. 2010, MNRAS, 404, 1425

Johnson, J. L., \& Bromm, V. 2006, MNRAS, 366, 247

Johnson, J. L., Dalla Vecchia, C., \& Khochfar, S. 2013, MNRAS, 428, 1857

Johnson, J. L., Greif, T. H., \& Bromm, V. 2007, ApJ, 665, 85

Johnson, J. L., Greif, T. H., Bromm, V., Klessen, R. S., \& Ippolito, J. 2009, MNRAS, 399, 37

Kaufmann, T., Wheeler, C., \& Bullock, J. S. 2007, MNRAS, 382, 1187

Klypin, A., Kravtsov, A. V., Valenzuela, O., \& Prada, F. 1999, ApJ, 522, 82

Komatsu, E., Smith, K. M., Dunkley, J., et al. 2011, ApJS, 192, 18

Koushiappas, S. M., Bullock, J. S., \& Dekel, A. 2004, MNRAS, 354, 292
Kravtsov, A. V. 2003, ApJL, 590, L1

Krumholz, M. R., \& Tan, J. C. 2007, ApJ, 654, 304

Kuhlen, M., \& Faucher-Giguère, C.-A. 2012, MNRAS, 423, 862

Labbé, I., González, V., Bouwens, R. J., et al. 2010, ApJL, 716, L103

Lehnert, M. D., Nesvadba, N. P. H., Cuby, J.-G., et al. 2010, Natur, 467, 940

Li, Y., Mac Low, M.-M., \& Klessen, R. S. 2005, ApJ, 626, 823

Lodato, G., \& Natarajan, P. 2006, MNRAS, 371, 1813

Loeb, A. 2010, How Did the First Stars and Galaxies Form? (Princeton, NJ: Princeton Univ. Press)

Loeb, A., \& Rybicki, G. B. 1999, ApJ, 524, 527

Lovell, M. R., Eke, V., Frenk, C. S., et al. 2012, MNRAS, 420, 2318

Machacek, M. E., Bryan, G. L., \& Abel, T. 2001, ApJ, 548, 509

Mac Low, M.-M., \& Ferrara, A. 1999, ApJ, 513, 142

Maio, U., Ciardi, B., Dolag, K., Tornatore, L., \& Khochfar, S. 2010, MNRAS, 407, 1003

Maio, U., Ciardi, B., Yoshida, N., Dolag, K., \& Tornatore, L. 2009, A\&A, 503,25

Maselli, A., Ciardi, B., \& Kanekar, A. 2009, MNRAS, 393, 171

Maselli, A., Ferrara, A., \& Ciardi, B. 2003, MNRAS, 345, 379

Mashchenko, S., Couchman, H. M. P., \& Wadsley, J. 2006, Natur, 442, 539

Mashchenko, S., Wadsley, J., \& Couchman, H. M. P. 2008, Sci, 319, 174

Mayer, L. 2010, AdAst, 2010, 278434

Mayer, L., Kazantzidis, S., Mastropietro, C., \& Wadsley, J. 2007, Natur, 445, 738

McKee, C. F., \& Ostriker, E. C. 2007, ARA\&A, 45, 565

Meiksin, A. A. 2009, RvMP, 81, 1405

Mellema, G., Iliev, I. T., Alvarez, M. A., \& Shapiro, P. R. 2006, NewA, 11, 374

Mesinger, A., Bryan, G. L., \& Haiman, Z. 2006, ApJ, 648, 835

Mihalas, D., \& Weibel Mihalas, B. 1984, Foundations of Radiation Hydrodynamics (New York: Oxford Univ. Press)

Moore, B., Ghigna, S., Governato, F., et al. 1999, ApJL, 524, L19

Muratov, A. L., Gnedin, O. Y., Gnedin, N. Y., \& Zemp, M. 2012, arXiv:1212.0909

Naoz, S., Noter, S., \& Barkana, R. 2006, MNRAS, 373, L98

Navarro, J. F., Eke, V. R., \& Frenk, C. S. 1996, MNRAS, 283, L72

Navarro, J. F., Frenk, C. S., \& White, S. D. M. 1997, ApJ, 490, 493

Oh, S. P. 1999, ApJ, 527, 16

Oh, S. P., \& Haiman, Z. 2002, ApJ, 569, 558

Oh, S. P., Haiman, Z., \& Rees, M. J. 2001, ApJ, 553, 73

Okamoto, T., Gao, L., \& Theuns, T. 2008, MNRAS, 390, 920

Ono, Y., Ouchi, M., Mobasher, B., et al. 2012, ApJ, 744, 83

O'shea, B. W., Abel, T., Whalen, D., \& Norman, M. L. 2005, ApJL, 628, L5

O'shea, B. W., \& Norman, M. L. 2007, ApJ, 654, 66

O'shea, B. W., \& Norman, M. L. 2008, ApJ, 673, 14

Panagia, N. 2005, in The Initial Mass Function 50 Years Later, Vol. 327, ed. E. Corbelli \& F. Palle (Dordrecht: Springer), 479

Pawlik, A. H., Milosavljević, M., \& Bromm, V. 2011, ApJ, 731, 54

Pawlik, A. H., \& Schaye, J. 2008, MNRAS, 389, 651

Pawlik, A. H., \& Schaye, J. 2011, MNRAS, 412, 1943

Petri, A., Ferrara, A., \& Salvaterra, R. 2012, MNRAS, 422, 1690

Prieto, J., Jimenez, R., \& Haiman, Z. 2013, arXiv:1301.5567

Raičević, M., Theuns, T., \& Lacey, C. 2011, MNRAS, 410, 775

Razoumov, A. O., \& Cardall, C. Y. 2005, MNRAS, 362, 1413

Razoumov, A. O., \& Sommer-Larsen, J. 2010, ApJ, 710, 1239

Regan, J. A., \& Haehnelt, M. G. 2009, MNRAS, 393, 858

Rhoads, J. E., Xu, C., Dawson, S., et al. 2004, ApJ, 611, 59

Ricotti, M. 2003, MNRAS, 344, 1237

Ricotti, M. 2010, AdAst, 2010, 271592

Ricotti, M., \& Gnedin, N. Y. 2005, ApJ, 629, 259

Ricotti, M., Gnedin, N. Y., \& Shull, J. M. 2001, ApJ, 560, 580

Ricotti, M., Gnedin, N. Y., \& Shull, J. M. 2002, ApJ, 575, 49

Ricotti, M., Gnedin, N. Y., \& Shull, J. M. 2008, ApJ, 685, 21

Ricotti, M., \& Ostriker, J. P. 2004, MNRAS, 352, 547

Robertson, B. E., Ellis, R. S., Dunlop, J. S., McLure, R. J., \& Stark, D. P. 2010, Natur, 468, 49

Romano-Díaz, E., Choi, J.-H., Shlosman, I., \& Trenti, M. 2011, ApJL, 738, L19

Roškar, R., Debattista, V. P., Brooks, A. M., et al. 2010, MNRAS, 408, 783

Rydberg, C.-E., Zackrisson, E., Lundqvist, P., \& Scott, P. 2013, MNRAS, 429, 3658

Safranek-Shrader, C., Agarwal, M., Federrath, C., et al. 2012, MNRAS, 426, 1159

Salpeter, E. E. 1955, ApJ, 121, 161

Salvadori, S., \& Ferrara, A. 2009, MNRAS, 395, L6

Santos, M. R. 2004, MNRAS, 349, 1137

Sawala, T., Scannapieco, C., \& White, S. 2012, MNRAS, 420, 1714

Sawicki, M., \& Thompson, D. 2006, ApJ, 648, 299 
Scannapieco, E., Ferrara, A., \& Madau, P. 2002, ApJ, 574, 590

Schaerer, D. 2003, A\&A, 397, 527

Schaye, J., \& Dalla Vecchia, C. 2008, MNRAS, 383, 1210

Schaye, J., Dalla Vecchia, C., Booth, C. M., et al. 2010, MNRAS, 402, 1536

Seljak, U., \& Zaldarriaga, M. 1996, ApJ, 469, 437

Shang, C., Bryan, G. L., \& Haiman, Z. 2010, MNRAS, 402, 1249

Shapiro, P. R., Giroux, M. L., \& Babul, A. 1994, ApJ, 427, 25

Shapiro, P. R., \& Kang, H. 1987, ApJ, 318, 32

Simcoe, R. A., Sullivan, P. W., Cooksey, K. L., et al. 2012, Natur, 492, 79

Springel, V. 2005, MNRAS, 364, 1105

Springel, V., White, S. D. M., Tormen, G., \& Kauffmann, G. 2001a, MNRAS, 328, 726

Springel, V., Yoshida, N., \& White, S. D. M. 2001b, NewA, 6, 79

Stacy, A., Bromm, V., \& Loeb, A. 2011, ApJL, 730, L1

Stiavelli, M., Fall, S. M., \& Panagia, N. 2004, ApJL, 610, L1

Stinson, G. S., Dalcanton, J. J., Quinn, T., Kaufmann, T., \& Wadsley, J. 2007, ApJ, 667, 170

Strigari, L. E., Bullock, J. S., Kaplinghat, M., et al. 2008, Natur, 454, 1096

Susa, H. 2007, ApJ, 659, 908

Tegmark, M., Silk, J., Rees, M. J., et al. 1997, ApJ, 474, 1

Thoul, A. A., \& Weinberg, D. H. 1996, ApJ, 465, 608

Toomre, A. 1964, ApJ, 139, 1217

Tornatore, L., Ferrara, A., \& Schneider, R. 2007, MNRAS, 382, 945

Trenti, M., Stiavelli, M., \& Michael Shull, J. 2009, ApJ, 700, 1672

Tseliakhovich, D., Barkana, R., \& Hirata, C. M. 2011, MNRAS, 418, 906

Tumlinson, J., Giroux, M. L., \& Shull, J. M. 2001, ApJL, 550, L1

Vera-Ciro, C. A., Helmi, A., Starkenburg, E., \& Breddels, M. A. 2013, MNRAS, 428,1696
Verhamme, A., Schaerer, D., Atek, H., \& Tapken, C. 2008, A\&A, 491, 89

Verner, D. A., Ferland, G. J., Korista, K. T., \& Yakovlev, D. G. 1996, ApJ, 465,487

Wang, J., Frenk, C. S., Navarro, J. F., Gao, L., \& Sawala, T. 2012, MNRAS, 424, 2715

White, S. D. M. 1996, in Cosmology and Large Scale Structure, ed. R. Schaeffer, J. Silk, M. Spiro, \& J. Zinn-Justin (Amsterdam: Elsevier), 349

Wiersma, R. P. C., Schaye, J., \& Smith, B. D. 2009, MNRAS, 393, 99

Wise, J. H., \& Abel, T. 2005, ApJ, 629, 615

Wise, J. H., \& Abel, T. 2007a, ApJ, 671, 1559

Wise, J. H., \& Abel, T. 2007b, ApJ, 665, 899

Wise, J. H., \& Abel, T. 2008a, ApJ, 684, 1

Wise, J. H., \& Abel, T. 2008b, ApJ, 685, 40

Wise, J. H., \& Cen, R. 2009, ApJ, 693, 984

Wise, J. H., Turk, M. J., \& Abel, T. 2008, ApJ, 682, 745

Wise, J. H., Turk, M. J., Norman, M. L., \& Abel, T. 2012, ApJ, 745, 50

Wolcott-Green, J., \& Haiman, Z. 2011, MNRAS, 412, 2603

Wolcott-Green, J., Haiman, Z., \& Bryan, G. L. 2011, MNRAS, 418, 838

Yajima, H., Choi, J.-H., \& Nagamine, K. 2011, MNRAS, 412, 411

Yajima, H., Umemura, M., Mori, M., \& Nakamoto, T. 2009, MNRAS, 398,715

Yoshida, N., Abel, T., Hernquist, L., \& Sugiyama, N. 2003, ApJ, 592, 645

Zackrisson, E., Rydberg, C.-E., Schaerer, D., Östlin, G., \& Tuli, M. 2011, ApJ, 740,13

Zackrisson, E., Zitrin, A., Trenti, M., et al. 2012, MNRAS, 427, 2212

Zeldovich, Y. B. 1970, A\&A, 5, 84

Zemp, M., Gnedin, O. Y., Gnedin, N. Y., \& Kravtsov, A. V. 2012, ApJ, 748,54 